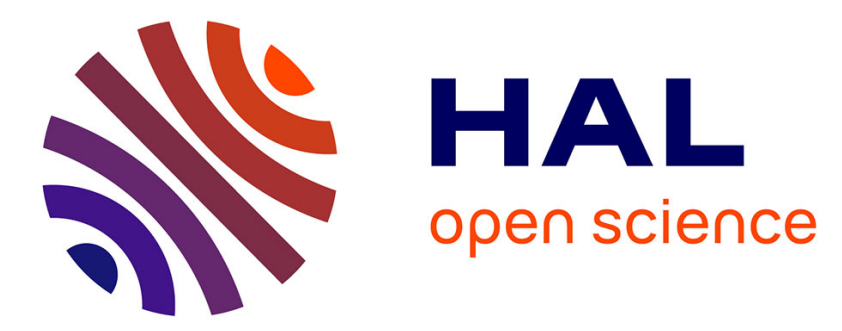

\title{
Les haches à douilles de type armoricain: une production strictement de l'âge du Fer. Critique des sources documentaires afférant à leur chronologie
} José Gomez de Soto

\section{- To cite this version:}

José Gomez de Soto. Les haches à douilles de type armoricain : une production strictement de l'âge du Fer. Critique des sources documentaires afférant à leur chronologie. Bulletin de la Société préhistorique française, 2015, 112 (1), pp.117-136. 10.3406/bspf.2015.14492 . hal-01137541

\section{HAL Id: hal-01137541 https://hal.science/hal-01137541}

Submitted on 30 Mar 2015

HAL is a multi-disciplinary open access archive for the deposit and dissemination of scientific research documents, whether they are published or not. The documents may come from teaching and research institutions in France or abroad, or from public or private research centers.
L'archive ouverte pluridisciplinaire HAL, est destinée au dépôt et à la diffusion de documents scientifiques de niveau recherche, publiés ou non, émanant des établissements d'enseignement et de recherche français ou étrangers, des laboratoires publics ou privés. 
Les haches à douilles de type armoricain: une production strictement de l'âge du Fer. Critique des sources documentaires afférant à leur chronologie

\begin{abstract}
Armorican type socketed axes: apurelyIron Age production. Critical analysis of the documentation on their chronology
\end{abstract}

José GOMEZ de SOTO

À la mémoire de Jacques Briard

\title{
Résumé
}

Dès le XVIII ${ }^{\mathrm{e}}$ siècle, les haches à douille en bronze dites de type armoricain, particulièrement abondantes en Bretagne et en Normandie, ont fait l'objet de débats, tant quant à leur finalité qu'à leur datation. Â la fin du XIX ${ }^{\mathrm{e}}$ siècle G. et A. de Mortillet affirmaient une datation à l'âge du Fer des modèles non fonctionnels de taille moyenne et miniature, les haches fonctionnelles du type de Brandivy restant attribuées au Bronze final. Mais c'est le point de vue de J. Déchelette qui l'emporta jusqu'à la fin du $X^{\mathrm{e}}$ siècle : ces haches étaient attribuée à l'horizon de l'épée en langue de carpe du Bronze final atlantique 3 (BF IIIb = LBA 3 britannique $=$ Ha B2-3, env. 950 à 800 av. J.-C.), avec une perduration au cours du premier âge du Ferreconnue par J. Briard.Bien qu'il commençât à être remis en cause à la fin du $\mathrm{XX}^{\mathrm{e}}$ siècle, ce point de vue traditionnel persiste chez certains auteurs. Aussi, le présent article se propose-t-il de produire un dossier aussi complet que possible de la question.

Les éléments démontrant une production de ces hachesau premier âge du Ferseulementsont indiscutables : sur le continent, aucune ne peut être datée antérieurement à la phase récente du premier âge du Fer(Ha D). L'actuelle «présence » de rares haches dans certains dépôts du BFa 3s'explique parleur pollution par des objets d'autres provenances, conséquences des aléas de leur conservation dans les collections et/ou les musées (ex., Plounéour-Lanvern, l'Île Verte, Aurigny). Parfois, quelques rassemblements d'objets aux provenances mal ou pas documentées réunis artificiellement ont été présentés commedesdépôts, lesquels n'avaient jamais existés (ex., Césarin). Dans d'autres cas, il s'agit d'une mélecture des sources documentaires (ex., Louvigné-du-Désert).Fait acquis essentiel, aucun dépôt de l'horizon de l'épée en langue de carpe, même parmi les plus volumineux (ex., Vénat, Jardin des Plantes, Prairie de Mauve, Belle Île), lorsqu'il est documenté de façon indiscutable, ne contient de hache à douille de type armoricain, aucune découverte récente non plus.

Des prototypes des haches à douille de type armoricain abondaient dans les dépôts du Bronze final atlantique 3, dont quelques haches ornées de nervures verticales d'origine britannique ouapparentées pouvant annoncer celles du type de Brandivy et plus généralement, avec leurs décors de baguettes verticales, les autres modèles de haches à douille de type armoricain. Surtout, les haches du type du Plainseau préfigureraient celles dutype duTréhou et des types proches de Dahouët et de Plurien. Au début du premier âge du Fer, des haches font effectivement figures d'intermédiaires entre celles du type du Plainseau et celles de la famille Tréhou/Dahouët/Plurien(dépôt du Fossé Creuzette à Verberie). Les haches de petite taille des types de Maure, Saint-James et Couville, en revanche, ne possèdent pas de prototypes dans les dépôts de la fin de l'âge du Bronze. Il pourrait s'agir d'une production récente, la fin d'un processus d'évolution conduisant aux formes les moins fonctionnelles, que ne pas connaître leur finalité exacte peut nous faire considérer, au même titre que celles en plomb presque pur, comme les plus aberrantes de toutes. 
S'il estdésormais acquis que la production des haches à douille de type armoricain n'avait pas encore commencé pendant le Bronze final,la question du début de leur fabrication reste incertaine : leurvisibilité en Gaule se limite au Ha D.Que cette production ait pu débuter dès l'étape ancienne du premier âge du Fer(Ha C)ne demeure, en l'état actuel de l'information, qu'hypothèse, comme aussi d'ailleursune fin de production ou du moins de dépôt au début du second.La multiplication des dépôts de ces haches est surtout une des manifestations du phénomène général de reprise en Gaule des enfouissements de dépôts de métalau cours du $\mathrm{Ha}$ $\mathrm{D}$, au même titre que, par exemple, les dépôts launaciens du Midi ou ceux de parures du Centre-Ouest et de la Loire moyenne.

\section{Mots clés}

Haches à douille de type armoricain, dépôts de bronze, parures, Bronze final atlantique 3, premier âge du Fer, Bretagne, Normandie

\section{Abstract (translated by E. Thauvin-Boulestin)}

The Armorican type socketed axes, particularly abounding in Brittany and Normandy, have been the subject of debates on both their purpose and their dating since the 18th century. At the end of the 19th century, G. and A. de Mortillet claimed an Iron Age attribution for some medium and miniature non-functional versions, whereas the Brandivy type functional axes were still dated to Late Bronze Age. Yet, this is J. Déchelette's point of view, confirmed by J. Briard in his famous book "Les dépôtsbretons et l'âge du Bronze atlantique" published in Rennes in 1965, which prevailed until the end of the 20th century: these axes were attributed to the carp's tongue sword horizon from Late Atlantic Bronze Age 3 (i.e. BFIIIb $=$ British LBA $3=\mathrm{HaB} 2-3$, around 950-800 BC). Even though J. Briard favoured a production of theses axes mostly during the end of the Bronze Age, he also demonstrated that this production continued during the Early Iron Age. This traditional point of view of a mostly Late Bronze Age production began to be questioned at the end of the 20th century, even by $\mathrm{J}$. Briard himself, nevertheless it still persists among some authors. The purpose of this article is thus to produce as comprehensive a file as possible on this issue. The elements testifying to a production of these axes exclusively during Early Iron Age are indisputable: on mainland Europe, no axe can today be dated for certain prior to $\mathrm{HaD}$. The rare axes currently appearing in some Late Bronze Age 3 hoards can be explained by the "pollution" of these hoards with objects from other origins, as a consequence of hazardous conservation conditions in collections and/or museums (for instance Plounéour-Lanvern and l'ÎleVerte, Finistère; Durtal, Maine-et-Loire; Longy-Common, Aurigny). Sometimes, objects from non- or poorly documented origins have been artificially brought together and presented as hoards, although the latter never existed (for instance Césarin, Corrèze). In other cases, it comes from a misinterpretation of the documentary sources (e.g.Louvigné-du-Désert, Ille-et-Vilaine). It is a crucial established fact that no hoard from the carp's tongue sword horizon among those formerly discovered and indisputably documented, even the largest ones (for instance Vénat, Jardin des Plantes, Prairie de Mauve), has ever yielded an Armorican type socketed axe. The same applies to all the hoards discovered during the second half of the 20th century and the beginning of the 21th (for instance Gouesnac'h, Finistère; Belle-Ile-en-Mer, Morbihan; SaintPère-en-Retz, Loire-Atlantique; Challans, Vendée; Meschers, Charente-Maritime; Triou, Deux-Sèvres). As a consequence, had these axes been already produced by the thousands at this period, it is absurd they should never appear in hoards, either complete or as fragments. 
Prototypes of Armorican type socketed axes were abundant during Late Atlantic Bronze Age 3: a few short axes decorated with vertical ribs, from British origins or related to them, could herald the Brandivy type axes, or more generally the other patterns of Armorican type socketed axes decorated with vertical ribs. Above all, axes of Plainseau type would prefigure those of Tréhou type and types close to the Dahouët and Plurien variants. At the beginning of Early Iron Age, some axes indeed appear as intermediate forms between the Plainseau type and the Tréhou/Dahouët/Plurien Armorican type ones (Fossé-Creuzette hoard in Verberie). On the contrary, the small axes of Maure, Saint-James and Couville types, as well as those made of almost pure lead, have no prototypes in Late Bronze Age hoards. They could represent a recent production of Armorican type socketed axes, in other words the end of an evolutionary process leading to the least functional forms, which, ignorant of their real purpose, we might consider the most absurd of all.

Undoubtedly, the production of Armorican type socketed axes had not yet begun during Late Bronze Age. Nevertheless, the question of their actual beginning remains uncertain: in Gaul, they all date from $\mathrm{HaD}$. Whether their production could have begun as early as Early Iron Age $(\mathrm{HaC})$ remains a mere hypothesis given our current state of knowledge, despite a few associations with axes of Sompting type in the British Isles, or in Brittany in the Hengoat hoard in Côtes-d'Armor: the production of Sompting type axes goes beyond the HaC Lynn Fawr horizon.

The increasing number of hoards of Armorican type socketed axes is above all one manifestation of a global phenomenon taking place in Gaul during the middle and late phases of Early Iron Age (HaD), the revival of burying of metal hoards, similar for instance to the hoards from Launac horizon in the South of France, or the ornament hoards in the CentreWest and Middle Loire area.

\section{Key words}

Armorican type socketed axes, bronze hoards, ornaments, Late Bonze Age 3, Early Iron Age, Brittany, Normandy

Les haches à douille en bronze dites « armoricaines », on le sait de longue date, isolées ou le plus souvent en dépôts parfois énormes, abondent en Bretagne et en Normandie, régions dans lesquelles leur production massive est une des plus originales de la Protohistoire. À l'expression «hache à douille armoricaine » devenue usuelle, nous préférerons celle de « hache à douille de type armoricain », sémantiquement plus exacte : une hache à douille armoricaine est une hache fabriquée ou trouvée en Armorique, quel qu'en soit le type ou l'époque, tandis que l'expression «de type armoricain» a valeur plus large, celle d'éponyme,et ne préjugenidu lieu de production, ni du lieu de trouvaille, ce dernier pouvant d'ailleurs se situer dans une province fort éloignée de l'Armorique.

\section{1. État de la question et justification de l'enquête}

Dès le début du XVIII ${ }^{\mathrm{e}}$ siècle, comme le rappela J. Briard (1965, p. 7 sq.), les haches à douille de type armoricain ont fait l'objet de débats, tant quant à leur finalité qu'à leur datation.

Dès 1881, G. et A. de Mortillet affirmaient la date tardive des haches à douille de type armoricain et ne les attribuaient qu'au seul âge du Fer (de Mortillet, 1881, pl. XCIII), à l'exception de celles du type de Brandivy, de grande taille et les seules fonctionnelles, qui se 
trouvaient données au Larnaudien, notre Bronze final (de Mortillet, 1881, pl. LXXV, $\mathrm{n}^{\circ}$ 788).L'argumentaire développé ne relevait que de l'intuition -qui se révélera exacte pour les haches non fonctionnelles - mais se ressentait fort du climat républicain et anticlérical de l'époque: «lorsqu'est arrivé le fer, la domination cléricale et l'orgueil aristocratique, qui s'appuient toujours sur le passé, se sont emparés de la hache de bronze pour en faire un objet votif, rituel et signe de distinction [...] ces haches de l'époque hallstattienne [...]» (de Mortillet, 1881, pl. XCIII).L'hypothèse d'une production centrée sur la fin de l'âge du Bronze n'en sera pas moins défendue par d'autres pionniers des études sur l'âge du Bronze, en particulier par J. Déchelette (1910a, p. 253) qui sera suivi par J. Briard(1965, p. 241 sq. ; 1995, p. 177 sq.) puis J. Rivallain $(1971,2012)$ :l'attribution chrono-culturelle retenue était l'horizon de l'épée en langue de carpedu Bronze final atlantique $3(\mathrm{BFa} 3=\mathrm{BF}$ IIIb $=\mathrm{LBA} 3$ britannique $=$ Ha B2-3, environ 950 à 800 av. J.-C.). Mais dès 1965, J. Briardadmit aussi une perduration de la production de ces haches au cours du premier âge du Fer.

La datation essentiellement au Bronze final atlantique 3avancée par J. Briardfera longtemps autorité auprès de la communauté des protohistoriens français et étrangers.Mais, et ce dès lesannées 90, J. Briard nuancerasérieusement son propos (Briard, 1991, p. 136 et 1995, p. 192) pour finalement rejeter définitivement le postulat d'une fabrication pendant la fin de l'âge du Bronze, et affirmer sans ambiguïté une production uniquement pendant le premier âge du Fer : «Les haches à douille de type armoricain sont un élément majeur du premier âge du Fer de Bretagne et de Normandie [....] elles sont rarement associées à d'autres objets sinon à des éléments du premier âge du Fer. Les associations anciennement signalées dans les dépôts en langue de carpe ne sont pas confirmées de nos jours, c'est pourquoi les haches à douille de type armoricain doivent être détachées de l'âge du Bronze et considérées comme une entité originale du début de l'âge du Fer » (Briard, 2001, p. 261).

J. Briard caressait le dessein de développer un ambitieux PCR afin de reprendre l'étude critique de ces haches.Il s'agissait non seulement de publier les nombreux dépôts découverts à partir des années $70 \mathrm{du} \mathrm{XX}^{\mathrm{e}}$ siècle restés inédits, mais aussi de produirela critique de la chronologie de ces instruments,ainsi que d'évaluer leur réelle diffusion en Europe, dégagée des attributions de provenances douteuses voire falsifiées (Briard, 2001, p. 262). Pour mettre en place ce PCR, J. Briard s'était assuré la collaboration d'une équipe internationale, mais, malheureusement, la cruelle maladie qui devait l'emporter lui interdit de lancer ce beau projet. La question revint à l'ordre du jour en 2005, lorsque la fouille du site de Kergariou à Quimper, Finistère, livra au fond d'une longue salle boisée enterrée un ensemble d'artefacts de bronze composé de haches à douille de type armoricain intactes et fragmentées associées à des débris d'objets divers, parmi lesquels deux fragments de parures annulaires de la fin du premier âge du Fer (Menez et al., 2005; Menez et Gomez de Soto, 2006). Cette étonnante trouvaille de Kergariou, d'un type inédit etbattant une nouvelle fois en brèche le point de vue traditionnel attribuant l'essentiel de la production des haches à douille de type armoricainau Bronze final atlantique 3,invita à reprendre dans le détail la question de la chronologie de ces instruments. Une présentation rapide des premiers résultats de l'enquête alors encore très incomplètefut donnée à l'occasion du colloque mixte AFEAF/APRAB tenu à Saint-Romainen-Gal en 2006 et résumée dans une courte note (Gomez de Soto, coord., 2009).

Cet ambitieux projet de $\mathrm{J}$. Briard, nul ne le reprit. Le présent article se propose, à titre de participation posthume et en hommage à la mémoire de ce grand chercheur, de présenter un dossier aussi complet que possible des éléments critiques permettant de réviser la situation chronologique traditionnellement assignée aux haches à douille de type armoricain.

\section{Dans les dépôts du Bronze final atlantique 3,pas de haches à douille de type armoricain}


Si on en fait l'analyse critique, les arguments autorisant à envisager le début de la production des haches à douille de type armoricain à la fin de l'âge du Bronze apparaissent des plus minces, car basés sur le contenu de dépôts de découverte ancienne mal documentée, ou qui paraissent avoir subi des mélanges dans les collections ou les musées depuis leur découverte.Unphénomène malheureusement fort répandu en ce qui concerne les découvertes anciennes, comme le rappelait encore voici peu $\mathrm{M}$. Talon à propos du dépôt du Bronze final atlantique 2 de Caix dans la Somme (Talon, 2012, p. 110), qui devrait inciter à une approche critique par remontée aux sources documentaires chaque fois que possible pour toute prise en compte de collection de découverte ancienne.

\subsection{Dépôts de Bretagne et des îles Anglo-Normandes}

- Plounéour-Lanvern, Finistère (Briard, 1965, p. 310, n² 225). La source princeps, publiée une quinzaine d'années après la découverte, situe le dépôt près du village de Kernivin, date la trouvaille de 1846 et précise qu'il ne comportait que sept objets, alors dans la collection Dorn à Quimper (Le Men, 1860). Ces objets sont actuellement conservés au musée de Châteaubriant, à l'exception d'un fragment de douille de pointe de lance ou de poignard, perdu ou non identifié (Vieau, 1976, p. 93 sq.). P. du Chatellier (1907, p. 277-278), de son côté, place ce dépôt à Keroberen, date sa découverte vers 1850 (ce qui reste à peu près compatible avec la date de 1846 donnée par R. G. Le Menn) et - ne faisant nulle allusion à ceux décrits par R. G. Le Men en 1860 - donne une liste de douze objets appartenant à sa collection. Au Musée d'Archéologie nationale à Saint-Germain-en-Laye, plusieurs de ces derniers font défaut, mais une hache à talon s'est curieusement ajoutée ${ }^{1}$. Ces deux séries viennent-elles bien d'un même dépôt qui en eût comporté au moins dix-neuf, R. G. Le Men n'ayant eu connaissance que d'une partie, ou s'agit-il de deux dépôts différents ? Surtout, l'ensemble tel qu'il se présente actuellement, offre un aspect très hétéroclite (on ne tiendra évidemment pas compte de la hache à talon surnuméraire de la collection du Chatellier conservée au MAN, non mentionnée par cet auteur) : une pointe de lance du type de Tréboul du début du Bronze moyen, trois haches à talon du type de Rosnoën du Bronze final atlantique 1 ou 2, deux pendants de harnais et des haches à douille du Bronze final atlantique 3 , deux haches à douille de type armoricain ;

- l'Île Verte à L'Île de Batz, Finistère (Briard, 1961 et 1965, p. 309, n 168). Découvert vers 1850 et très mal documenté, le dépôt présente actuellement l'aspect d'un ensemble hétéroclite mêlant objets du Bronze moyen (fig. $1, \mathrm{n}^{\circ}$ 5), du Bronze final atlantique 3 (fig. $1, \mathrm{n}^{\circ} 1$ à 4, 6, 8, 10 à 15), une hache du type de Sompting ou apparentée (fig. $1, \mathrm{n}^{\circ}$ 7) et une hache à douille du type armoricain de Couville (fig. 1, n 9). Déjà, en 1883, V. Micault (cité par J. Briard, 1961, p. 44) soupçonnait des mélanges ;

- le bourg de Kerlouan, Finistère. Découvert en 1925, il ne semble pas avoir fait l'objet de publication détaillée avant sa mention par J. Briard quarante ans plus tard (Briard, 1965, p. $309, \mathrm{n}^{\circ}$ 175). L'exactitude de la provenance de la hache à douille de type armoricain qu'il est censé contenir reste donc sujette à caution ;

- Plélan-le-Grand, Ille-et-Vilaine (Briard, 1965, p. 313, n 317). La fiabilité de la composition de ce dépôt sera mise en doute par J. Briard lui-même (1991, p. 141), qui ne retiendra plus la hache du type de Couville ;

- la Moussaye à Plénée-Jugon, Côtes-d'Armor. Les bracelets du Bronze final que le dépôt était censé contenir ne sont qu'un rajout postérieur à la découverte, comme l'indique clairement J. Briard (1965, p. 275) ;

- Louvigné-du-Désert, Ille-et-Vilaine (Briard, 1965, p. 313, n 308). La publication princeps (Chapelet, 1909), figure une hache à douille du type du Plainseau et précise que les deux 
autres haches du dépôt sont analogues : ce dépôt ne contiendrait donc pas de haches à douille de type armoricain ;

- carrière de Rungallic à Collorec, Finistère (Giot, 1954 ; Briard, 1965, p. 275 et 308, n 138). Un dépôt de haches à douille et un autre de bracelets en or furent découverts, le premier «dans un creux de rocher ", l'autre «à environ 1,50 $\mathrm{m}$ du point de la précédente trouvaille [...] à la surface du rocher » (Giot, 1954) : les deux ensembles ne sont donc pas strictement associés, même si l'on ne peut exclure que l'enfouissement sur le même terrain put n'être pas fortuit et témoigner d'une tradition transmise au cours du temps, un phénomène illustré sur d'autres sites. Quoi qu'il en soit, il est abusif de considérer que l'un date l'autre. Qui plus est, la datation proposée pour les bracelets, de forme très peu caractéristique, est assez imprécise : Bronze moyen ou Bronze final selon C. Eluère (1982, p. 88, 151 et 259), mais une plus récente encore ne saurait être rejetée et parait même d'autant plus probable que les deux marques en V frappées sur l'un d'eux (Giot, 1954, p. 57) seraient pour le moins inhabituelles pour ne pas dire aberrantes pour des bijoux de l'âge du Bronze armoricain ;

- la Pierre du Villain à Longy Common, Aurigny. Ce dépôt découvert en 1833 ne fut publié que dix ans après sa découverte, mais avec un inventaire très détaillé (Lukis, 1843, p. 9-10). Des publications plus complètes ne parurent qu'au $\mathrm{XX}^{\mathrm{e}}$ siècle, alors que le dépôt semblait avoir déjà connu des ajouts.Lahache à douille du type de Couville attribuée au dépôtn'apparaît pas avant les publications du XX $\mathrm{XX}^{\mathrm{e}}$ siècle (Kendrick, 1928, p. 62 sq. et pl. VIIVIII ; Jockenhövel, 1980, pl. 88, n $^{\circ} 29$ ), aussi sa provenance réelle pose-t-elle problème : Lukis, très précis dans ses descriptions de 1843, n'eût pas manqué de mentionner un objet aussi original, ce qui n'est pas le cas. De plus, un fragment de rebord de situle de type tessinois du premier âge du Fer, du modèle de celle du cimetière du Rocher au Bono dans le Morbihan (Milcent, 1993), se trouve réuni avec les objets du dépôt, ce qui contribue encore à jeter un sérieux doute quant à son homogénéité tel qu'il se présente de nos jours, visiblement pollué par des trouvailles extérieures ;

- les Grandes Fosses du domaine de Mainlands, Saint Lawrence Valley, Jersey. Découvert en 1871 dans des conditions non précisées, ce dépôt n'a connu de publication, d'ailleurs sommaire, que soixante-cinq ans après sa découverte (Hawkes, 1937, p. 106 sq.) : ces soixante-cinq ans laissaient largement le temps pour des mélanges.

\subsection{Autres dépôtsde France}

- château de Durtal, Maine-et-Loire. Ce dépôt du Bronze final atlantique 3 trouvé en 1860, qui aurait été énorme (on a parlé d'un volume de $1 \mathrm{~m}^{3}$, ce qui paraît peu crédible), est très mal documenté : on ne possède pas à son sujet d'inventaire suivant de près sa découverte, et le nombre des objets subsistantsconservés au musée Saint-Jean à Angersaugmenteselon les auteurssans qu'on sache pourquoi de 1868 à 1921 (Cordier, 2009, p. 272). Tel que présenté par G. Cordier et M. Gruet (1975, p. 189 et fig. 36 à 38) puis par G. Cordier seul (2009, p. 272), ce nombre serait de trente-sept, parmi lesquels quatre haches à douille de type armoricain (trois intactes et un fragment), mais aussi quatre haches du Bronze moyen. En sus des doutes instillés par la contradiction des sources documentaires, onne peut que s'interroger quant à la fiabilité de ces associations : ce dépôt du Bronze final atlantique 3 possèderait, pour ce qui en subsisterait, $10,81 \%$ de haches à douille de type armoricain, mais également 10,81 $\%$ de haches du Bronze moyen, autrement dit, les deux types de haches en des proportions anormalement élevéespour un dépôt du Bronze final atlantique 3. La collection du musée d'Angers paraît plus un rassemblement hétéroclite d'objets de provenances diverses que constituée des véritables épaves d'un dépôt de la fin de l'âge du Bronze; d'ailleurs, les patines permettent de classer les bronzes en trois ensembles... qui curieusement coïncident 
avec chacun des trois groupes d'objets, celui du Bronze moyen, celui du Bronze final et celui des haches à douille de type armoricain ;

- Vénat à Saint-Yrieix, Charente. Les troishaches à douille de type armoricain figurées par R. Audin et R. Riquet $\left(1956, \mathrm{n}^{\circ} 4,8,9\right)$ se trouvaient mélangées avec les objets de provenance authentique au musée de la Société archéologique et historique de la Charente à Angoulême : le prouvent sans ambiguïté les publications princeps du dépôt, réalisées aussitôt faitela découverte par des chercheurs renommés qui n'en font nullement état (Favraud, 1893; George et Chauvet, 1894) et les catalogues du musée qui indiquent ces pièces, l'une sans provenance, les deux autres de Vendée (Catalogue..., 1885, p. 26 ; Idem, 1915, p. 70, D1, 78). Des tentatives de vente de haches à douille du type de Couville frauduleusement attribuées au dépôt avaient été déjouées dès la fin du XIX ${ }^{\mathrm{e}}$ siècle (Gomez de Soto, 1980, p. 87) ;

- Césarin à «Tulle », Corrèze (Roussot-Larroque, 1984). Ce supposé dépôt composé de deux haches à talon du Bronze moyen, d'une herminette à douille du Bronze final et de deux haches à douille de type armoricain, conservé au musée Saint-Raymond à Toulouse, est totalement inconnu de la bibliographie ancienne, en particulier de l'appendice du Manuel de J. Déchelette (1910b). Les patines de trois des pièces décrites par J. Roussot-Larroque sont très différentes, vert clair pour l'une des haches à talon, noirâtre pour l'autre, verte avec des altérations en écailles pour une des haches à douille ; d'autre part uncontrôle au musée SaintRaymondconfirme que les cinq objets possèdent tous des patines différentes, celles des deux haches à douille n'étant quant à elles pas même analogues ! Ce prétendu « dépôt » paraît bien n'être lui aussi que le rassemblement artificiel d'objets de provenances hétérogènes. Qui plus est, le toponyme Césarin n'existe pas à Tulle, mais à Naves, selon M.-B. Chardenoux et J.-C. Courtois (1979), qui d'ailleurs dans leur inventaire des haches du Midi de la France ne présentent pas ces objets comme associés.

\subsection{Bilan de l'examen critique}

Si l'on prend en compte l'ensemble des dépôts du Bronze final atlantique 3 de Bretagne et de Normandie, on ne peut que constater la faible proportion de ceux un temps présumés contenir des haches à douille de type armoricain:

- Bretagne historique (actuelle région Bretagne et département de Loire-Atlantique) : sur 57 dépôts inventoriés par J. Briard (1965, p. 199-201), seuls cinqétaient retenus par cet auteur comme en ayant possédé(Briard, 1965, p. 275), quatre seulement en retirant celui de Plélanle-Grand ensuite contesté (Briard, 1991), soit 8,7 \% d'entre eux. Cette proportion ne tombe plus qu'à 5,3\% si l'on actualise le calcul à partir de l'inventaire donné par P.-Y. Milcent (2012, p. 218 sq.), qui porte le nombre des dépôts à 94. Pour chacun des cinq dépôts, le nombre de haches à douille de type armoricain aurait d'ailleurs été on ne peut plus modeste : deux à Plounéour-Lanvernet à Louvigné-du-Désert, une seule à l'Île Verte, au bourg de Kerlouan et à Plélan-le-Grand, soit un total de seulement sept instruments sur plusieurs centaines d'objets ! On constate aussi qu'aucun des grands dépôts tels ceux à Nantes du Jardin des Plantes (Briard, 1971) et de la Pairie de Mauves (Briard et al., 1966) ne sont présumés en contenir. Avec plus de 900 objets extrêmement variés, dont certains très originaux, le dépôt de Keriéro à Bangor, Belle-Île, Morbihan, qui ne contient aucune hache à douille de type armoricain ou fragment (Boulud et Fily, 2009, p. 290), est particulièrement emblématique de cet état de fait ! Les cartes de répartition des dépôts du Bronze final atlantique 3 et des dépôts de haches à douille de type armoricain se superposent largement, comme l'a de longue date montré J. Briard (1957, p. 324-325) et le rappelle J.Rivallain(2012, p. 154) : si la production de ces haches avait été au moins en partie contemporaine des dépôts de l'horizon de l'épée en langue de carpe, il est évident que ces derniers en contiendraient, ce qui n'est pas le cas ; 
- Normandie : 38dépôts répertoriés, maisaucun ne contient de hache à douille de type armoricain (Milcent, 2012, p. 218-238).

La supposée présence de haches à douille de type armoricain dans certains dépôts de l'horizon de l'épée en langue de carpe du Bronze final atlantique 3 peut donc relever de deux cas de figure :

- des pollutions par des objets d'autres provenances, conséquences des aléas de leur conservation dans les collections et/ou les musées, qu'un retour aux sources, lorsque celles-ci sont suffisamment précises, permet de détecter, comme nous le montrâmes plus haut: un phénomène de perte d'identité des objets suivi d'attributions fallacieuses, trop classique conséquence d'une chaîne de négligences comme le rappelaient opportunément A. Letor (1999) pour les haches à douille de type armoricain prétendument trouvées en Belgique, ou I. Stead (1998, p. 127 sq.) à proposde remarquablesobjets de l'âge du Fer présumés découverts dans les îles Britanniques, mais n'en venant pas ;

- des mélectures des sources documentaires.

Une autre source d'erreur est le rassemblementaccidentelou artificiel d'objets aux provenances mal ou pas documentées, formant in fine un pseudo-ensemble qui n'avait jamais existé.

Exista-t-ilmalgré tout quelques authentiques «dépôts bric-à-brac» réunissant bronzes de récupération et haches à douille de type armoricain ? Il faut le souligner à nouveau, les plus hétéroclites, en particulier ceux de Plounéour-Lanvern, de l'Île Verte et de Durtal, sont particulièrement mal documentés, voire absolument pas comme celui dit de Césarin, et de ce fait, leur composition paraît on ne peut plus suspecte : la réalité de tels dépôts dans le monde atlantique continental reste donc à démontrer. Nous y reviendronsinfra.

Pour terminer, reste à souligner qu'aucun des grands dépôts de la fin de l'âge du Bronze du Nord, du Nord-Ouest et de l'Ouest de la France, de découverte ancienne bien documentée comme ceux de la Prairie de Mauves à Nantes, de Longeville ou de Vénat à Saint-Yrieix, ou de trouvaille récente dûment contrôlée tels ceux de Gouesnac'h, Belle-Ile-en-Mer, Saint-Pèreen-Retz, Challans, Meschers ou Triou, n'a livré de hache à douille de type armoricain. Aussi, on comprendrait mal que, si celles-ci se fussent déjà fabriquées à l'époque - qui plus està milliers d'exemplaires ! - elles n'y figurassent jamais, entières ou fragmentées. Comme nous l'avons rappelé ci-dessus, J. Briard avait déjà souligné, lors du colloque consacré à l'âge du Bronze atlantique tenu à Beynac en 1990, le constat de cette récurrente absenceà propos des dépôts alors récemment découverts en Armorique (Briard, 1991, p. 136), position qu'il réaffirma encore plus clairement dix ans plus tard (Briard, 2001, p. 261).

\subsection{Des bronzes de récupération dans des dépôts de haches à douille de type armoricain}

J. Briard (1965, p. 271) mentionne encore cinq dépôts de haches à douille de type armoricain qui contiendraient des bronzes nettement plus anciens, en l'occurrence des haches à talon, mais un seul paraît résister à l'analyse critique des sources documentaires :

- celui d'Ergué-Ermel, Finistère, au lieu de découverte précis inconnu, aurait été composé de haches à talon et de haches à douille, mais le type de ces dernières n'est pas précisé (du Chatellier, 1907, p. 329);

- la composition de celui de Kervellou à Ploaré, Finistère, est de fait inconnue : P. du Chatellier (1907, p. 260) indique une « cachette de fondeur qui contenait en tout 31 haches à talon ou (c'est nous qui soulignons) à douille », cité par J. Déchelette (1910b, n 268, p. 45), qui écrit « 31 haches, les unes à t., les autres à d. »;

- celui de Parc-ar-Puns à Plobannalec, Finistère, n'est connu qu'à travers un article non illustré de la presse locale, qui plus est paru une vingtaine d'années après la découverte (Oges, 1952), donnant l'inventaire suivant : une hache à talon, une « à douille quadrangulaire » (vu le 
manque de précision, l'appartenance au type du Plainseau est tout aussi recevable que celle au type armoricain, surtout si la masse indiquée pour cet instrument, $350 \mathrm{~g}$, est exacte), un lingot plano-convexe entier et cinq fragments. Cet ensemble paraît plutôt un classique dépôt de l'horizon de l'épée en langue de carpe ;

- le «dépôt » de Vaux-sur-Aure, Calvados, est en réalité l'amalgame de deux dépôts, l'un de haches à talon, l'autre de haches à douille, découverts respectivement en 1863 et 1875 sur le territoire de la même commune (Verney, 1994, p. 33) ;

- en revanche, publié dès sa découverte (du Chatellier, 1891, p. 23), le dépôt de Saint-Honoré à Plogastel-Saint-Germain, Finistère, qui réunit une hache à talon et onze à douille de type armoricain, parait fiable. C'est bien le seul !

Quant aux dépôts de hache à douille découverts depuis 1970, aucun ne contient d'objets de date antérieure, ni hache à talon, ni artefact du Bronze final atlantique 3 (Briard, 2001 ; Rivallain, 2012, cédérom) $)^{2}$.

\section{Les haches à douille de type armoricain en contexte de l'âge du Fer}

Les associations vérifiables démontrant un enfouissement postérieur à l'âge du Bronze des haches à douille de typearmoricain, dont un certain nombre furent déjà remarquées par $\mathrm{J}$. Briard dès 1965, sont en revanche en nombre appréciable.

\subsection{Dépôts dans des situles en métal ou en céramique}

Les exemples sont peu nombreux :

- la situle en bronze du premier âge du Fer ayant contenu le dépôt de Spézet, Finistère (Briard, 1965, p. 244);

- les vases en céramique aux formes inspirées des situles en bronze du type de Kurd ayant contenu les dépôts de Roudouallec à Kerhon, Morbihan, et surtout celui de Mahalon à Bogoudonou, Finistère (Briard, 1965, p. 244 ; Briard et al., 1982-83, p. 52). Sur la façade atlantique, en Saintonge par exemple (Marchadier, 2005), de tels vases n'apparaissent pas avant le $\mathrm{Ha} \mathrm{C}$ récent ;

\subsection{Parures ou fragments de parures du premier âge du Fer moyen et récent (Ha D) dans des dépôts bretons de haches à douille de type armoricain}

J. Briard(1965, p. 271-275) avait remarqué des bracelets du premier âge du Fer dans plusieurs dépôts de haches à douille de type armoricain, ceux de Coat-jou-Glas à Plonéis, Finistère (fig. 2, $n^{\circ}$ 1-2), de Rest-an-Cornou à Bourbiac et de Saint-Bugan à Loudéac (fig. 2, no 3), Côtesd'Armor. En revanche, le bracelet du dépôt de Guesman au Tréhou,réduit àunsimple fragment de barre atypique (Briard, 1965, fig. 104, n $^{\circ}$ 4), n'est pas un bracelet à bossettes comme il sera écrit plus tard par erreur (Briard, 1995, p. 193).

Depuis, d'autres dépôts ont révélé des parures ou fragments de parures du premier âge du Fer :

- un des dépôts deLocoal-Mendon, Morbihan(Rivallain, 2012, fig. 77), contiendrait, à côté de fragments de bracelets peu caractéristiques (fig. 3, $\mathrm{n}^{\circ \mathrm{s}} 2-4$ ), des fragments d'un ou plusieurs bracelets à bossettes massives (fig. $3, \mathrm{n}^{\circ} 1$ ). Le modèle de ce(s) bracelet(s) à bossettes est analogueà celui de deux exemplaires de la nécropole du Ha D de Trévent à La Cambe, Calvados (Verney, 1993, fig. 3 et 4);

- le dépôt du Torvet à Trelly, Manche (fig. 2, nos 6 à 12), associe des fragments de bracelets du Ha D1, dont de deux à petites bossettes qui ne sont pas sans évoquerceux de Kergariou à 
Quimper dont il sera fait état ci-dessous, une petite parure annulaire terminée par deux disques et divers petits objets (Verney, 1999 ; Aranda et al., 2013) ;

- le dépôt de la Forgerais à Ruffigné, Loire-Atlantique (L'Helgouach, 1985 et 1999; Philippe, 1992) contient un ove creux venant d'un bracelet ou plutôt d'anneau de cheville du Ha D1, accompagné d'un fragment de barre, possible vestige d'un bracelet atypique (fig. 2, $\left.\mathrm{n}^{\circ \mathrm{s}} 4-5\right)$;

- dans un petit dépôt trouvé aux environs de Quimper, une hache à douille de type armoricain accompagnait deux bracelets, décrits mais non figurés dans la publication princeps (Giot, 1954). L'un d'eux est présenté " [...] formé d'une tige pleine à section ronde, de $6 \mathrm{~mm}$ d'épaisseur moyenne. Mais, aux extrémités qui s'amincissent, sont soudées, du côté extérieur, deux volumineuses sphères creuses de bronze, de $23 \mathrm{~mm}$ de diamètre, dont une seule est restée intacte » (Giot, 1954, p. 59). J. Gachina (1975), afin de le comparer aux bracelets d'une tombe de Port-d'Envaux en Charente-Maritime, a donné un dessin de ce bracelet d'après un document des archives de P.-R. Giot (fig. 2, n 13). D'autres bracelets du même modèle, mais aux boules plus petites, apparaissent dans diverses tombes du Calvados: tombe 20 de la Campagne à Basly (San Juan, 1999 ; San Juan et Le Goff, 2003), Vaucelles à Caen (Verney, 1993, fig. 2, n 8), sépulture 2010 de la Fosse Touzé à Courseulles-sur-Mer, (Jahier, 1999), sépulture 2086 de la ZAC Objectif's Sud d'Iffs, nécropole du Mesnil à Verson (Giraud et Nivez, 2014). Des bracelets du type Bb.4.XVI.a du Ha D1-2 de France centrale présentent une construction proche (Milcent, 2004, fig. 83, $\mathrm{n}^{\circ}$ 8). Les bracelets de Port-d'Envaux présentent, comme celui des environs de Quimper et les exemplaires normands, des boules fixées non axialement sur la tige comme le seraient des tampons, mais orthogonalement à elle. Le bracelet de Basly se trouvait associé à quatre anneaux de cheville ouverts à oves creux, donnant une date au Ha D1, celui de Courseulles-sur-Mer serait à peine plus tardif que ce dernier. Un bracelet du type Port-d'Envaux provient d'une double sépulture masculine et féminine de Biganos en Gironde, dans laquelle il se trouvait associé à une épée à antennes à poignée à plaquettes de type aquitain (Dautant et al., 1984). La période de productionde ces épées, ou du moins de leur utilisation, s'étend jusqu'au tout début du second âge du Fer : une se trouve associée à une fibule du début de La Tène A ancienne dans une tombe de Lauzeré à Fourques-sur-Garonne, Lot-et-Garonne (Béhague et al., 2006 ; Béhague et Martineau, 2011). Le parallélisme stylistique entre le bracelet du dépôt des environs de Quimper et les bracelets de type Port-d'Envaux amène à soulever l'hypothèse d'une possible date tardive pour le premier et partant, pour sinon la production, du moins l'enfouissement jusqu'au tout début du second âge du Fer, des dernières haches à douille de type armoricain. Nous y reviendrons plus loin.

\subsection{L'ensemble métallique de Kergariou à Quimper}

L'ensemble métallique de Kergariou à Quimper provient d'une structure excavée creusée sur un habitat de l'âge du Fer (Menez et al., 2005; Menez et Gomez de Soto, 2006). À l'exception d'une hache trouvée dans son comblement, il se trouvait réparti entre une petite fosse creusée au fond de la structure et ses abords immédiats. À côté des quatre haches intactes (cinq en comptant celle issue du comblement de la structure), toutes du type du Tréhou (fig. 4, $\mathrm{n}^{\circ \mathrm{s}} 1-2$ ), il montre, par rapport à la norme des dépôts de haches à douille de type armoricain - les petits fragments de haches et autres débris de métal ne s'y trouvent qu'en nombre restreint (Briard, 1965, p. 271 et 1972) - une surreprésentation de débris de haches de divers types (47 au total ; ex., fig. $4, \mathrm{n}^{\circ \mathrm{s}} 3$ à 5 ), dont du type de Couville, et autres fragments d'objets (10 en tout; ex., fig. $4, \mathrm{n}^{\circ \mathrm{s}} 6$ à 9), dont deux débris de parures annulaires (fig. 4, $\mathrm{n}^{\circ \mathrm{s}} 8$-9).Sadisposition sur le terrain a peu à voir avec les conditions habituelles des 
dépôts classiques. Aussi peut-on peut s'interroger quant à qualifier cetensemble de dépôt : peut-être s'agit-il derestes d'un fonds d'atelier rejetés ou abandonnés ?

Les fragments de parures indiquent clairement une datation dans la phase récente du premier âge du Fer, au Ha. D et probablement plus précisément au Ha D2-3. Une des deux parures pourrait être considérée, soit comme un modèle archaïque par rapport à celles des sépultures armoricaines (Milcent, 1993), normandes (Verney, 1993) ou de Gaule de l'Est (Chaume, 2001, p. 132 sq.), soit plutôt comme un prototype des modèles de la fin du Ha D/La Tène A ancienne.

Les rares tessons datables fournis par la structure sont attribuables à la fin du premier âge du Fer (fig. 4, $\mathrm{n}^{\mathrm{os}} 10$ à 14), date encore confirmée par un fragment d'anneau décoré en lignite (fig. $4, \mathrm{n}^{\circ} 15$ ) analogue aux deux quiaccompagnent l'inhumation du Ha D du tumulus 7 de la nécropole du Rocher au Bono dans le Morbihan (Milcent, 1993, p. 29 et pl. 3).

\subsection{Une hache du type de Sompting dans le dépôt deHengoat, Côtes-d'Armor ?}

Le dépôt du Moulin de Bizienà Hengoat, composé de haches du type du Tréhou, contenait deux fragments jointifsd'une même hache à douille de type non armoricain (Menez et Fily, 2009 ; Rivallain, 2012, p. 150), attribuable au type de Sompting ou à un modèle apparenté (fig. 5). Cette association n'est pas sans évoquer le «dépôt» britannique de Danebury, Hantshire,Angleterre, dont nous traiterons infra.

Une autrehache à douille de modèle apparenté au type de Sompting figureraitencore dans le dépôt de l'île Verte à L'île de Batz (fig. 1, n 7), dépôt dontla fiabilité, comme nous l'avons $\mathrm{vu}$, est discutable.

\subsection{Des haches à douille du type de Couville en contexte launacien}

Des haches à douille du type de Couville, authentiques et/ou imitations locales, figurent dans plusieurs dépôts launaciens du Languedoc :Launac, environs de Carcassonne, Montpellier, Murviel-lès-Béziers, La Croix de Mus (Guilaine, 1969 et 1972 ; Chardenoux et Courtois, 1979, p. 119 ; Laroche et al., 2008, p. 49 ; Verger, 2013). Le dépôt de Bautarès contient une petite hache, variante du type de Maure (Laroche etal., 2008, p. 50).

\subsection{Le problématique « dépôt » de Danebury, Hantshire}

Le « dépôt » de Danebury (Cunliffe, O’Connor, 1979 ; Jockenhövel, 1980, pl. 100 ; ici, fig. 6), associe une hache du type du Tréhou (fig. 6, $\mathrm{n}^{\circ} 11$ ) à onze autres bronzes dont les dates s'étalent largement dans le temps, de l'horizon d'Areton Down au premier âge du Fer.À cette dernière période appartiennent deux rasoirs (fig. 6, $\mathrm{n}^{\circ \mathrm{s}}$ 6-7)respectivement des types de Gramat et de Feldkirch/Bernissart (Jockenhövel, 1980) et deux haches à douille du type de Sompting (fig. 6, nos 8-9).Cet ensemble hétéroclite n'est pas un douteux fonds de musée ou de collection privée, mais bien une découverte dûment contrôlée. Est-ce un véritable dépôt dispersé par les racines et les animaux fouisseurs comme le présument B. Cunliffe et B. O'Connor,et qui seraitalors un petit ensemble du type «dépôt de Salisbury » (Stead, 1998 ; Cooneyet al., 2011), ou le résultat d'une suite d'apports successifs en un même espace bénéficiant d'une forte charge symbolique ou rituelle, à l'exemple de certains dépôts britanniques en milieu humide (Coombs, 1992) ? Quoi qu'il en soit, les leçons essentielles en sont d'une partqu'il ne peut s'agir d'un dépôt du Bronze final atlantique 3 (horizon britannique d'Ewart Park), d'autre part la possibleassociation d'une hache à douille de type armoricain avec des bronzes du premier âge du Fer ancien (Ha C). Nous y reviendronsinfra. 


\subsection{Apports de quelques recherches récentes}

Enfin, des recherches récentes confirment l'indépendance des dépôts de haches à douille de type armoricain par rapport aux contextes de la fin de l'âge du Bronze :

- la position des lieux d'enfouissement des dépôts du Bronze final et de ceux des dépôts de haches à douille de type armoricain dans le Centre-Ouest de la Bretagne et le Finistère ne paraissent pas obéir aux mêmes logiques topographiquesd'enfouissement (Fily, 2008) ;

- le métal des objets du Bronze final atlantique 3 et celui des haches à douille de type armoricain venant de la même région montrent des compositions chimiques différentes et exclusives les unes des autres (Le Carlier et al., 2009 ; Le Carlier et al., 2011). Mais aussi, pour le dépôt de Trelly, hachesà douille de type armoricain et parures de types du Ha D associées présentent des compositions tout à fait compatibles, ce qui suggère leur contemporanéité (Aranda et al., 2013, p. 117).

\section{Pour certains dépôts hétéroclites : le modèle « Salisbury Hoard » ?}

L'attribution sans discussion à l'horizon de l'épée en langue de carpedu Bronze final atlantique 3 de ces dépôts hétérogènes ne tenait que sur un présupposé : les haches à douille de type armoricain dateraient pour leur très grande majorité de la fin de l'âge du Bronze. Or il faut le souligner - la composition de ces ensembles hétéroclites ne correspond en rien aux normes des dépôts de l'horizon de l'épée en langue de carpe : lorsque ceux-ci, ce qui déjà est rare, contiennent des objets plus anciens, ces derniers n'y figurent qu'en très faible proportion, comme des trouvailles récentes telles celles de la Tiédenaie à Saint-Père-en-Retz, Loire-Atlantique (Maggi et Paitier,1994) ou de Kergaradec à Gouesnac'h, Finistère (Fily, 2009) permettent de le vérifier ${ }^{3}$.

L'hypothèse que certains de ces « dépôts bric-à-brac » fussent authentiques, c'est-à-dire pas le seul produit de mélanges postérieurs à leur découverte, ne peut cependant être systématiquement rejetée sans autre forme de procès : il faut ici tenir compte de la leçon de l'étonnant dépôt anglais de Netherhampton, Wiltshire, dit «dépôt de Salisbury »(Stead, 1998 ; Cooneyet al., 2011). Constitué de plus de cinq-cents objets,ce dépôt réunit des pièces produites sur une très longue période, pendant presque tout l'âge du Bronze et l'âge du Fer, qui se rattachent aux horizons métalliques d'Arreton Down, Acton Park, Taunton, Penard, Willburton, Ewart Park, Llyn Fawr, etc., les plus récents datant du second âge du Fer vers 200 à 30 avant J.-C. On peut interpréter cette variété comme une accumulation d'objets sur la longue durée, ou comme la découverte suivie de leur réenfouissement de divers dépôts alors identifiés comme sacrés et complétés parde nouveaux apports après leur trouvaille. Cette accumulation sur la longue durée neserait pas sans rappeler les dépôts dans des lieux cultuels en milieu humide de Flag Fen et Fengate Power Station, étalés du début du Bronze final britannique (horizon de Penard) à la période romaine (Coombs, 1992).

Rien n'interdit d'envisager que des ensembles du type dépôtde Salisbury, associant des objets métalliques d'époques variées, aient pu aussi se trouver réunis en Armorique ou ailleurs en Gaule. La récente découverte du dépôt de Roussier à La Motte-en-Champsaur dans les Hautes-Alpes (Barge et Borel, 2007 ; Barge, 2011, p. 16 sq.)indique en effet qu'une telle pratique put être épisodiquement en usage sur le continent. Cet ensemble associe un dépôt homogène du Bronze final III à un petit lot d'objets en fer de La Tène finale voire du début de la période romaine ${ }^{4}$ : nous avons ici visiblement affaire à un dépôt du Bronze final retrouvé à date tardive et qui,respecté car sans doute reconnu comme sacré, fut réenfoui de concert avec des objets de l'époque de sa découverte.

Quoi qu'il en soit de la possibilité de réenfouissements tardifs de bronzes de récupération voire d'entiers dépôts de l'âge du Bronze découverts au cours de l'âge du Fer, la véritable 
date d'ensevelissement de ces éventuels dépôts à composition hétéroclite contenant des haches à douille de type armoricain ne peut évidemment être donnée que par leurs objets les plus récents, qui fournissent le terminus post quem de leur enfouissement. En l'occurrence, ce seraient les haches à douille de type armoricain, dont tout désormais indique qu'elles ne furent produites que pendant l'âge du Fer. Mais rappelons aussi qu'aucun des dépôts hétéroclites présenté comme contenant des haches à douille de type armoricain de France n'est fiable...

\section{Les dates ${ }^{14} \mathrm{C}$}

Deux dates ${ }^{14} \mathrm{C}$ seulement sont disponibles :

La première est fournie par un foyer semblant associé au dépôt de Saint-Bugan à Loudéac, Côtes-d'Armor : GsY 42, 2519 +/- 130 ans (Briard, 1965, p. 275).

Date calibrée (avec le logiciel CALIB REV5.0.2) :

- à $1 \sigma(68,3 \%$ de certitude) : entre 799 et 508 cal. BC et entre 438 et 420 cal. BC ;

- à $2 \sigma(95,4 \%$ de certitude) : entre 968 et 964 cal. BC, entre 929 et $362 \mathrm{cal}$. BC, entre 268 et 265 cal. BC.

Les fourchettes de probabilité données par la calibration à $2 \sigma$ correspondent, la première à la fin du Bronze final atlantique $2(=\mathrm{BF}$ IIIa/Ha B1), la seconde à une large période allant du Bronze final atlantique 3à La Tène $\mathrm{B}$, la dernière à la fin de La Tène $\mathrm{B}$. Si l'on se satisfait de la fourchette à $1 \sigma$, les deux plages de probabilité se trouvent entièrement calées sur les âges du Fer.

La seconde date a été obtenue à partir d'une pièce de bois utilisée dans le dispositif d'enfouissement du dépôt Marchéssieux, Manche : Ly. 2676, 2740 +/- 100 ans (Tabbagh et Verron, 1983, p. 383).

Date calibrée (avec le logiciel CALIB REV5.0.2) :

- à $1 \sigma(68,3 \%$ de certitude $)$ : entre 1002 et 806 cal. BC ;

- à $2 \sigma(95,4 \%$ de certitude) : entre 1254 et 1238 cal. BC, entre 1214 et 755 cal. BC, entre 684 et $669 \mathrm{cal}$. BC, entre 608 et $600 \mathrm{cal}$. BC.

Les deux premières plages de probabilités de la calibration à $2 \sigma$, séparées par un court espace de 40 ans, s'étalent du Bronze final atlantique 1 (= BF Ib/BzD2) au début du premier âge du Fer (Ha $\mathrm{C} 1)$, et les deux dernières recouvrent la phase récente du premier âge du Fer $(\mathrm{Ha} \mathrm{D})$. Ces dates, aux fourchettes trop larges, manquent par trop de précision, mais on soulignera qu'elles recouvrent très largement le premier âge du Fer, et qu'elles ne peuvent nullement être invoquées pour affirmer une date du Bronze final de la production des haches à douille de type armoricain.

\section{De l'origine des haches à douille de type armoricain}

Entre la fin de l'âge du Bronze vers 800 av. J.-C. et le début de l'étape récente du premier âge du Fer(Ha D) vers 640 av. J.-C., c'est-à-dire pendant le premier âge du Fer ancien (Ha C), aucune découverte de hache à douille de type armoricain n'est attestée en France, ou du moins, ne peut être reconnue avec certitude faute d'association indiscutable avec d'autres modèles d'artefacts bien datés. La seule association présentée comme telle, à Bordeaux, Gironde(Roussot-Larroque, 2013, p. 72), serait celled'une hache du type de Couville avec un rasoir du type de Quarante (Jockenhövel, 1980, $\mathrm{n}^{\circ}$ 695).Le contrôle de la source princepsqui indique «un dé romain [c'est nous qui soulignons], une hache armoricaine et une pendeloque [il s'agit du rasoir, cf. Roussot-Larroque, 1982, p. 33] trouvés en faisant une tranchée entre les rues Tête-Nègre et Hérard-Dubreuilh » (Bardié et Dosque, 1898-1899, p. 23) montre que cette présumée association est pour le moins incertaine, pour ne pas dire douteuse. Elle est si 
discutable que l'hypothèse que le rasoir - alors non réuni avec la hache - provînt d'une sépulture fut même envisagée(Roussot-Larroque, 2005, p. 90)...

L'absence de hache à douille de type armoricain pendant le $\mathrm{Ha} \mathrm{C}$ en Bretagne et en Normandie comme ailleurs en France est-elle réelle, ou qu'une illusion, la conséquence du quasi abandon de la pratique des enfouissements de dépôts de métal pendant cette période, contrairement à ce qui se passait dans les îles Britanniques avec ceux de l'horizon de Llyn Fawr? Sur le continent, en Gaule, les dépôts ne réapparaîtront avec une réelle importance que pendant le Ha D (Milcent, 2004, carte p. 193), avec en particulier les dépôts launaciens à composition complexedu Languedoc (Guilaine, 1972; Verger, 2013) et ceux largement constituésde parures du Centre-Ouest septentrional (Tauvel, 1974 ; Gomez de Soto, Milcent, 2000), du Bassin parisien (Piette, 1989 ; Villes, 1995, p. 14 et 130-131 ; Chevrier, 2007), du Quercy (Arnal et al., 1969; Gascó, 2013) et de l'Est du Massif central (Millotte, 1972 ; Milcent, 2013a et b) .

Pourtant, après 800 av. J.-C., la production d'objets fonctionnels d'usage couranten bronze s'est poursuivie longtemps en Europe nord-alpine pendant tout le premier âge du Fer ancien $(\mathrm{Ha} \mathrm{C})$ et moyen (Ha D1), voire au-delà, avec une typologie quipar rapport à celle la fin de l'âge du Bronzen'a évolué que lentement, voire pour certains outils, pas,contrastant avec celle de l'armement, en particulier celle des épées, qui évoluait plus rapidement.En témoignent, pour nous limiter à quelques exemples, des dépôts tels ceux de Wattenheim/Alsenborn, Kr. Kaiserlautern, en Sarre (Kolling 1968, pl. 54-55 ; Kibbert, 1984, pl. 98-99) ou de Scharlachkopf à Bingen, Kr. Mainz-Bingen, en Rhénanie-Palatinat (Kibbert, 1984, pl. 108), ceux de l'horizon britannique de Llyn Faw (O'Connor, 2007), ou encore la hache à douille de la tombelle 3 de Court-Saint-Etienne en Belgique (Mariën, 1958, p. 108 sq.). Sans doute en est-il allé de même au cours du premier âge du Fer en Gaule de l'Ouest, mais là, l'absence de dépôtspendant cette période comme le petit nombre des bronzes abandonnés sur les habitats nous privent d'informations suffisantes quant à la production métallique courante.Dans d'autres régions de Gaule,la productiond'outils fonctionnels et d'armes en bronze de typologie analogue ou très comparable à celle du Bronze final se poursuivit jusque pendant leHa D, comme l'indiquent, pour nous limiter encore à quelques exemples, une hache à douille du dépôt de la Mouleyre à Saint-Pierre-d'Eynac, Haute-Loire (Millotte, 1972 ; Milcent, 2004, pl. 83-84), ou en Gaule du Sud, comme la lance et son talon de la tombe 149 de la nécropole du Peyrou à Agde (Nickels, 1989, p. 227 et fig. 191) et les pointes de lances et des outils des dépôts launaciens (Guilaine, 1972 ; Chardenoux et Courtois, 1979, pl. 91-92 ; Verger, 2013).

Des haches à douille à section quadrangulaire existent tôt dans les îles Britanniques, dès l'horizon de Bishopsland, parallèle à l'horizon de Rosnoën du Bronze final atlantique 1 français (= BF Ib-IIa / BzD2-HaA1), par exemple dans le dépôt éponyme (Rowlands, 1976, pl. 34 ; Jockenhövel, 1975, fig. 11; O’Connor, 1980, fig. 37-38). Mais vu le décalage temporel, il paraît imprudent d'y reconnaitre l'origine des haches à douille de type armoricain de l'âge du Fer.

Sur le continent et dans les îles Britanniques, les haches du type du Plainseau, haches à douille à section quadrangulaire et ouverture souvent subquadrangulaire, les plus nombreuses des haches à douille dans les dépôts du Bronze final atlantique 3 sur le continent, sont de longue date admises comme vraisemblables prototypes des haches à douille de type armoricain, mais ce ne sont pas les seuls possibles. En particulier, dans plusieurs dépôts français figurent des haches à douille relativement trapues ornées de nervures terminées ou non par des globules (Briard, 1965 ; Eluère, 1979; Coffyn et al.,1981, carte 5), dont, en ajoutant celles des îles Anglo-Normandes, près d'une vingtaine d'exemplaires du type sudgallois ou apparentées (Burgess, 2012) ${ }^{6}$. Ce décor de nervures n'est pas sans évoquer celui des haches du type de Brandivy,présumées les plus anciennes des haches à douille de type 
armoricain car encore fonctionnelles.Ces nervuresseretrouventaussi régulièrement sur leshaches de taille moyenne ou miniature (Briard, 1965 ; Rivallain, 2012, p. 92 sq.). On connaît des haches d'origine ou d'inspiration britannique dans plusieurs dépôtscontinentaux du Bronze final atlantique 3. Pour ne citer que quelques-uns de ces derniers : la Tiédenaie à Saint-Père-en-Retz, Loire-Atlantique (Maggi et Paitier, 1994, pl. 3, n 65), Challans, Vendée (Verney, 1990, fig. 6, $\mathrm{n}^{\circ}$ 16), les Bois à Lutz-en-Dunois, Eure-et-Loir (Cordier, 2009, fig. $225, \mathrm{n}^{\circ}$ 2), Vénat à Saint-Yrieix, Charente (Coffyn et al.,1981, pl. 20, $\mathrm{n}^{\circ} 1$ ). Ces haches d'inspiration britannique des dépôts du Bronze final continentalsont toutefois nettement plus courtes que celles du type de Brandivy dont la longueur varie de 130 à $160 \mathrm{~mm}$ pour une moyenne de 145,8 mm (Briard, 1965, p. 249) : par exemple, seulement 109 mm à Saint-Pèreen-Retz, $108 \mathrm{~mm}$ à Challans, $104 \mathrm{~mm}$ à Lutz-en-Dunois et à Vénat.La plupart des haches du type sud-gallois mesurent, quant à elles,environ 110 à 150 mm (Briard et Verron, 1976 ; Burgess, 2012, p. 245).

Datable du tout début du premier âge du Fer par une bouterolle naviforme de fourreau d'épée proche des plus anciens modèles du premier âge du Fer, le dépôt du Fossé Creuzette à Verberie, Oise (Blanchet, 2001), contient encore des haches du type du Plainseau, mais certaines autres, bien qu'encore très proches, s'en éloignent déjà et pourraient annoncer celles de type armoricain (Milcent, 2012, p. 145).

Dans les îles Britanniques, l'horizon de Llyn Fawr connaît aussi des productions de haches à douille proches de celles des types du Tréhou et apparentés. Ces instruments,généralement ornés de baguettes verticales, sont désignés sous l'appellation de type de Sompting, qui, comme le type armoricain, se décline en une certaine variété de modèles (O'Connor, 2007).Elles peuvent se trouver associées dans quelques dépôts à des haches de type armoricain(Milcent, 2012, p. 163-165).Une hache de ce type ou apparentée figure, nous l'avons vu supra, dans le dépôt de Hengoat, Côtes-d'Armor (fig. 5). Dans le dit « dépôt » de Danebury, évoqué supra (Cunliffe, O’Connor, 1979), se trouvent deux haches du type de Sompting et une du type du Tréhou, ainsi que deux rasoirs du Ha C, mais l'ensemble, nous l'avons vu, pose problème. Si l'association est bien réelle, nous voulons dire au sens d'unenfouissement au même moment et non échelonné dans le temps, elle pourrait peut-être signifier une apparition des haches à douille de type armoricain - du moins de celles du type du Tréhou - dès la phase ancienne du premier âge du Fer. Dans cette hypothèse, devra-t-on pour autant aller jusqu'à suggérer une origine britannique pour ces dernières ? Il va sans dire que ces deux hardies hypothèses devront se voir confirmées par d'autres découvertes...D'autant plus que, comme le souligne P.-Y. Milcent (2012, p. 165), la fourchette chronologique des haches du type de Sompting déborde 1'horizon de Llyn Fawr, c'est-à-dire s'étale aussi sur le premier âge du Fer récent (Ha D).

En l'état actuel de l'information, la zone d'origine des premières haches à douille de type armoricainreste donc incertaine, et leur production dès le Ha $\mathrm{C}$ conjecturale : les seules certitudes les rapportent au Ha D.

Le phénomène de production de haches à douille non fonctionnelles au cours du Ha $\mathrm{D}$ ne fut d'ailleurs pas limité, on le sait de longue date, aux seules Armorique et Normandie. Outre les productions britanniques, on connait bien celles de l'horizon launacien en Gaule du Sud (Guilaine, 1972, p. 345 sq. ; Briard et Verron, 1976, p. 89). Un phénomène du même ordre, mais moins intense, concerna également les régions de la Meuse et du Rhin inférieur en Allemagne et aux Pays Bas, avec en particulier les haches à douille du type de Geistingen (Postmaet al., 2005 ; Warmenbol, 2012-2013).

\section{La fin des haches à douille de type armoricain}


La trouvaille de Keralio à Quimper indique une production des haches à douille de type armoricain jusque pendant la phase récente du premier âge du Fer.

À propos du bracelet du dépôt des environs de Quimper, nous avons envisagé la possibilité d'une date d'enfouissement tardive, au tout début de La Tène A ancienne.

De fait, le seul exemple de hache à douille de type armoricain dans un contexte protohistoriquepostérieur au premier âge du Fer est celui d'unedu typedu Tréhou incluse dans l'ensemble 800, de La Tène ancienne, de l'établissement de Keralio à Pont-l'Abbé, Finistère, un site occupé depuis le VI ${ }^{\mathrm{e}}$ s. av. J.-C. (Hinguant et al., 1998, p. 91 et fig. 42). Mais on pourrait objecter qu'elle n'y était que résiduelle, voire même qu'elle y fut transportée depuis un proche site du premier âge du Fer, comme habitat deKerarthur (Roy, 2000).

L'absence de ces haches dans le comblement ou sur le sol des souterrains de Bretagne (Giot et al., 1995, p. $286 s q$.) constitue encore un indice de l'abandon de la production de ces artefacts dès avant la période laténienne.

\section{Discussion et conclusion}

Les éléments démontrant une production tardive des haches à douille de type armoricain sont éloquents : sur le continent, aucun dépôtni en Armorique,ni en Normandie, ni ailleurs, ne peut être actuellement daté antérieurement au $\mathrm{Ha} \mathrm{D}$. Ce constat s'impose par les données disponibles, et nul n'est besoin pour la conforter de retenir quelques associations incertaines de Bretagne et d'Anjou qui pourtant,elles aussi,iraient dans le sens d'une production postérieure au Bronze final :

- le présumé dépôt de haches à douille et d'un bracelet à bossettes de Carfantain à Dol-deBretagne, Ille-et-Vilaine. Cette association, bien que déjà mise en doute par J. Briard (1965, p. 275), se voit reprisepar J. Rivallain (2012, p. 147). Nous ignorons l'origine de cette prétendue association: les sources (Lavallée, 1888 et 1891 ; Robert, 1898, p. 63) ne la mentionnent nullement ;

- le regroupement de haches à douille, de céramiques laténiennes et de lingots de fer du type Spitzbarren de Kerolzec à Saint-Martin-des-Champs, Finistère, rejeté par J. Briard(1965, p. 275);

- le présumé ensemble d'anneaux à bossettes, anneaux à oves creux et haches à douille censé venir de la région de Saumur en Maine-et-Loire (Mongellaz et Meuret, 1999) : dans leur article consacré aux âges du Bronze et du Fer en Anjou, G. Cordier et M. Gruet (1975, p. 231 et 227) ne les associent pas.

Cependant, la question du début de la fabrication de ces haches reste posée : visibilité pendant le seul Ha D ne signifie pas nécessairement production pendant ce seul Ha D. L'ensemble de bronzes de Danebury - que nous hésitons à qualifier de dépôt stricto sensu - pourrait suggérer un début de production dès le $\mathrm{Ha} \mathrm{C}$, hypothèse qui demande à être étayée par de nouveaux éléments.

Mais, fait acquis et désormais indiscutable, aucun dépôt de l'horizon de l'épée en langue de carpe, même parmi les plus volumineux, lorsqu'il est documenté de façon indiscutable, ne contient de hache à douille de type armoricain : on peut en tenir pour certain que la production de ces haches n'avait pas encore commencé pendant le Bronze final.

D'autre part, aucun dépôt de l'horizon de l'épée en langue de carpe du continent, contrairement à ce qu'on a souvent écrit, ne contient de manière indubitable d'objets caractéristiques du Ha C.Il en a ététraité en détail dans un récent article (Gomez de Soto, 2014),inutile de reprendre ici en détail les cas des présumées associations les plus souvent avancées, en particulier :le rasoir de type hallstattien attribué au dépôt de l'île Guennoc à Landéda, Finistère (Briard, 1965, p. 219 et 239 ; Briard et Onnée, 1996) ${ }^{7}$ :une découverte indépendante du dépôt (du Chatellier, 1891 ; Cartailhac, 1891) ;undébris de languette d'épée 
hallstattiennedu dépôt du Petit Villatte à Neuvy-sur-Barangeon, Cher (Cordier, 1996, fig. 6, $\mathrm{n}^{\circ} 8$ et 2009, fig. 236, $\mathrm{n}^{\circ} 8$ ) : un fragment d'objetbrut de fonte de type indéterminé (Milcent, 2012, note 355, p. 176) ; les fragment de lames d'épéesde la famille de Gündlingen du dépôt de Vénat à Saint-Yrieix (Gomez de Soto, 1984) et du dépôt de Chamery, Marne (Doize, 1965) :des fragments de lame d'épée du type d'Ewart Park; etc. Il est évident que des objets caractéristiques, en particulier rasoirs ou fragments d'épées de la famille de Gündlingen, ne sauraient manquer de figurer dans des dépôts de l'horizon de l'épée en langue de carpe qui eussent été enfouis au cours du $\mathrm{Ha} \mathrm{C}$, puisque ces instruments et ces armes étaient largement utilisés en Gaule de l'Ouest au cours de cette période (Jockenhövel, 1980 ; Verger, 1999; Roussot-Larroque, 2013). D'ailleurs, si l'on admettait- alors que tout démontre le contraire (Gomez de Soto, 2014) - que sur le continent les tout derniers dépôts de l'horizon de l'épée en langue de carpe furent enfouis au tout début du $\mathrm{Ha} \mathrm{C}$, ce serait un argument supplémentaire pour démontrer, s'il en était encore besoin, que la production des haches à douille de type armoricain n'avait pas encore débuté, puisqu'aucun n'en contient !

Que le début de la production des haches à douille de type armoricain débutât au cours du Ha $\mathrm{C}$ ne demeure donc, en l'état actuel de l'information, qu'une hypothèse. Quant à la fin de cette production, elle dut coïncider avec la fin du premier âge du Fer, c'est-à-dire s'étaler jusque pendant la première moitié du Ve siècle av. J.-C. Quelquesabandons purent encore avoir eu lieu audébut du second âge du Fer, mais le seul exemple sûrement répertorié, celui de Keralio à Pont-l'Abbé, reste d'interprétation discutable.

\section{Remerciements}

L'auteur remercie chaleureusement Yves Menez, adjoint du conservateur régional de l'Archéologie de Bretagne,responsable de la fouille du site de Kergariou,qui lui en a confié l'étude du dépôt métallique, étude à l'origine de l'enquête présentée ici.

Il remercie également ses collègues, qui par leurs informations ou avis, l'ont aidé dans sa recherche :Muriel Fily, archéologue départementale du Finistère, et à nouveau Yves Menez, pour leurs informations sur le dépôt de Hengoat ;Brendan O'Connor, du Bronze Age Study Group, pour ses informations sur les dépôts d'Aurigny et de Jersey ;Pierre-Yves Milcent, maître de conférences à l'université de Toulouse-Le Mirail, pour les informations concernant les bronzes attribués au dépôt de Durtal et ceux dits de Césarin ; Muriel Fily, à nouveau, pour ses renseignements concernant des dépôts de haches à douille récemment découverts en Bretagne ; Sylvie Boulud-Gazo, maître de conférences à l'université de Nantes, pour ses informations au sujet des fragments de parures de Forgerais, et Marie-Hélène Santrot, conservatrice au Musée Dobrée à Nantes, pour l'envoi de leurs photographies et l'autorisation de les reproduire ; Anne-Françoise Chérel, chargée d'études à l'Inrap, pour ses informations sur les sépultures de la ZAC Objectif's Sud à Iffs ; B

runo Boulestin, de l'UMR 5199-PACEA, pour les calibrations des dates ${ }^{14} \mathrm{C}$; CatherineLe Gall, bibliothécaire du laboratoire Préhistoire et Archéosciencesà l'université de Rennes 1, qui lui a procuré les données bibliographiques qui lui faisaient défaut.

\section{Notes}

1. Les mélanges sont nombreux au MAN à Saint-Germain-en-Laye dans la collection du Chatellier : par exemple, le « dépôt » de La Guerche, présumé du BFa 3 (Briard et al., 1986), qui se présente actuellement comme un mélange hétéroclite : la «pointe de Palmela » est certainement une pointe de flèche de Méditerranée orientale ; le bracelet 2, du type dit de 
Bignan, date du Bronze moyen 2 ; le bracelet 3 est par son décors d'ocelles proche d'un de la sépulture 43/16 de l'âge du Fer de Saint-Jean-Trolimon, Finistère, ainsi que de certaines productions suisses du début de La Tène ; les bracelets 4 et 6 , atypiques, peuvent dater du $\mathrm{BFa}$ 3 ; le bracelet 5 est un anneau de situle du BFa 3 ou du Ha C. Donc, seuls les objets 4 à 6 viennent peut-être du dépôt, s'il datait bien du BFa 3. Ces mélanges dans la collection du Chatellier se sont produits on ne sait quand : ses collections étaient bien classées, les mélanges sont probablement posthumes.

2. Et informations Muriel Fily pour diverses découvertes récentes encore inédites.

3. Nous serons plus circonspects quant au dépôt du Bronze final atlantique 3 de Tourtenay, Deux-Sèvres, qui aurait contenu une hache à talon intacte et deux autres fragmentaires (Germond, Champême, 1998, p. 258), découverte clandestine avec un détecteur de métaux revendue à un amateur, qui curieusement affirma qu'on la lui avait volée... lorsqu'il fut question de réexaminer certains objets (information GeorgesGermond).

4. Il n'y a pas lieu ici de discuter des datations proposées par l'auteur, trop tardive pour les bronzes qu'elle place possiblement au début du Hallstatt, trop ancienne pour les objets de fer attribués au début de La Tène. Nous remercions notre collègue Jean-Paul Guillaumet, directeur de recherche émérite au CNRS, pour son avis quant aux objets en fer.

5. Le terme horizon de Lynn Fawr est pris ici dans la large acception britannique, non dans la plus restreinte proposée par Pierre-Yves Milcent (2012, p. 165).

6. Les haches à douille du Bronze final ornées de nervures verticales sont largement répandues en Europe hors des îles Britanniques, jusqu'en Pologne par exemple (Baron et al., 2014).

7. Contrairement à une affirmation récente, le dépôt de l'île Guennoc (Roussot-Larroque, 2013, p. 73) ne contient pas de hache à douille de type armoricain.

\section{Références bibliographiques}

ARANDA B., LE CARLIER de VESLUD C., MARCIGNY C., LE BANNIER J.-C. (2013) Le dépôt de haches à douille de type armoricain du Hallstatt D1-D2 de Trelly (Manche). Interprétations à partir des analyses chimiques élémentaires, Bulletin de la Société préhistorique française, 110, 1, p. 105-119.

ARNAL J., COUCHARD L., LORBLANCHET M. (1969) - La grotte de Roucadour (Thémines, Lot), Archivode PrehistoriaLevantina, 12, p. 55-91.

AUDIN R., RIQUET R. (1956) - Saint-Yrieix «Vénat », Charente, France, Inventariaarchaeologica (F 6), Anvers, 6 feuillets.

BARDIE A., DOSQUE R. (1898-1899) - Compte-rendu de la séance du 18 novembre 1899, Bulletin dela Société archéologique de Bordeaux, 23, p. XLIX. 
BARGE H. (2011) - Cachettes et dépôts sacrés dans les Alpes du Sud. Dépôts métalliques de l'Âge du Fer, Theix, ActiliaMultimedia.

BARGE H., BOREL B. (2007) - Un dépôt de La Tène au lieu-dit Roussier (La Motte-enChampsaur, Hautes-Alpes), in P. Barral, A. Daubigney, C. Dunning, G. Kaenel, M.-J. Roulière-Lambert (dir.), L'âge du Fer dans l'arc jurassien et ses marges. Dépôts, lieux sacrés et territorialité à l'âge du Fer, actes du XXIXe colloque international de l'AFEAF (Bienne, 5-8 mai 2005), Besançon, Presses universitaires de Franche-Comté, p. 681-698.

BARON J., MIAZGA B., NOWAK K. (2014) - Functions and contexts of Bronze Age metal casting mouldsfromPoland, Bulletin de la Société préhistorique française, 111, p. 325-338).

BEHAGUE B., MARTINEAU P., PROUIN Y. (2006) - Fourques-sur-Garonne, Lauzeré, BilanscientifiqueAquitaine, Paris, Ministère de la Culture et de la Communication, p. 130131.

BEHAGUE B., MARTINEAU P. (2011) - Les sépultures de la fin du premier âge du Fer de Fourques-sur-Garonne (550-400 av. J.-C.), in A. Colin, C. Sireix, F. Verdin (dir.), Gaulois d'Aquitaine, Bordeaux, Musée d'Aquitaine, p. 94-96.

BLANCHET J.-C. (2001) - Nouveaux dépôts de la transition Âge du Bronze / Âge du Fer dans le contexte de la moyenne vallée de l'Oise. Du monde des chasseurs à celui desmétallurgistes. Hommage scientifique à la mémoire de Jean L'Helgouac'h et mélanges offerts à J. Briard, Rennes, Revue archéologique de l'Ouest (suppl. 9), p. 171-180.

BOULUD S., FILY M. (2009) - Les dépôts métalliques de l'extrême fin du Bronze final en Bretagne: nouvelle évaluation des données à la lumière des découvertes récentes, in M.-J. Roulière-Lambert, A. Daubigney, P.-Y. Milcent, M. Talon, J. Vital (dir.), De l'âge du Bronze à l'âge du Fer en Europe occidentale (Xe - VIIe siècle av. J.-C.). La moyenne vallée du Rhône aux âges $d u$ Fer, actes du colloque international mixte AFEAF et Association pour la Promotion de l'âge du Bronze (Saint-Romain-en-Gal, 26-28 mai 2006), Dijon (Revue archéologique de l'Est, suppl. 27), p. 283-298.

BRIARD J. (1957) - Le Bronze de faciès atlantique en Armorique, Congrès préhistorique de France. Compte-rendu de la XV Session. Poitiers - Angoulême 15-22 juillet 1956, Paris, Société préhistorique française, p. 313-327.

BRIARD J. (1961) - Le dépôt de l'Ile Verte en l'île de Batz, Finistère, Travaux du Laboratoired'Anthropologie préhistorique, Rennes, p. 44-48.

BRIARD J. (1965) - Les dépôts bretons et l'Âge du Bronze atlantique, Rennes, Laboratoire d'Anthropologie préhistorique, $353 \mathrm{p}$.

BRIARD J. (1971) - Un dépôt de l'âge du Bronze Final : le Jardin des Planches à Nantes (Loire-Atlantique), Études préhistoriques et protohistoriques Pays de la Loire, 2, p. 3-35.

BRIARD J. (1972) - Un dépôt du Bronze final: les haches à douille armoricaines de Treillières (Loire-Atlantique). Congrès préhistorique de France, compte-rendu de la XIXe session,Auvergne, 1989, p. 117-126. 
BRIARD J. (1991) - Le groupe de l'épée en langue de carpe en Armorique : une révision, in C. Chevillot et A. Coffyn (dir.), L'âge du Bronze atlantique, actes de colloque (Beynac, sept. 1990), Périgueux, Vesuna, p. 125-144.

BRIARD J. (1995) - L’Âge du Bronze, Protohistoire de la Bretagne, Rennes, Ouest-France, p. 25-198.

BRIARD J. (2001) - Les haches à douille de type armoricain, in J. Briard, J. Gomez de Soto, P.-Y. Milcent, J.-P. Pautreau, Les recherches sur l'âge du Bronze en Poitou-Charentes, Centre, Pays de Loire, Bretagne et Basse Normandie, Documents d'Archéologie méridionale, 24, p. 261-262.

BRIARD J., ELUERE C., MOHEN J.-P., VERRON G. (1982-83) - Mission au British Museum: objets de l'âge du Bronze trouvés en France. I - Les ensembles, Antiquités nationales, 14-15, p. 24-58.

BRIARD J., avec coll. GOULETQUER P.-L. et ONNEE Y. (1966) - Dépôts de l'âge du Bronze en Bretagne. La Prairie de Mauves, Rennes, Travaux du Laboratoire d'Anthropologie préhistorique, $44 \mathrm{p}$.

BRIARD J., LECERF Y., LE ROUX C.-T., MEURET J.-C., ONNEE Y., BOURHIS J.-R. (1986) - L'âge du Bronze dans la région de La Guerche (Ille-et-Vilaine), Revue archéologique de l'Ouest, 3, p. 59-78.

BRIARD J., ONNEE Y. (1996) - L'âge du Bronze à l'île Guennoc. Landéda, Finistère, AssociationManche Atlantique pour la recherche archéologique dans les îles, 9, p. 31-41.

BRIARD J., VERRON G. (1976) - Typologie des objets de l'Âge du Bronze en France. IV : Haches (2)Herminettes, Paris, Société préhistorique française, 90 p.

BURGESS C. (2012) - South Welsh Socketed Axes and OtherCarp's Tongue Conundrums, in W. J. Britnell et R. J. Silvester (dir.), Reflections on the Past. Essays in Honor of Frances Lynch, Welshpool, Cambrian Archaeological Association, p. 237-253.

CARTAILHAC E. (1891) - Rasoir en bronze du Finistère, L'Anthropologie, 2, p. 400.

Catalogue (1885) - Catalogue du musée archéologique d'Angoulême, Bulletin de la Société archéologique et historique de la Charente, $5^{\mathrm{e}}$ série, VII, p. 3-70.

Catalogue (1915) - Catalogue du musée de la Société archéologique et historique de la Charente, Angoulême, Société archéologique et historique, 174 p.

CHARDENOUX M.-B., COURTOIS J.-C. (1979) - Les haches dans la France méridionale, Münich, C.H. Beck (PrähistorischeBronzefunde, IX, 11), 187 p.

CHAPELET H. (1909) - Cachette d'objets en bronze à Louvigné-du-Désert (Ille-et-Vilaine), L'Hommepréhistorique, 7, p. 53-55. 
CHATELLIER P. du (1891) - De quelques cachettes découvertes dans le Finistère, L'Anthropologie, 2, p. 17-24.

CHATELLIER P. du (1907) - Les époques préhistoriques et antiques dans le Finistère, $2^{\mathrm{e}}$ éd., Rennes et Quimper, Plihon et Hommay, 391 p.

CHAUME B. (2001) - Vix et son territoire à l'Âge du Fer. Fouilles du mont Lassois et environnement du site princier, Montagnac, M. Mergoil (Protohistoire européenne, 6), 643 p.

CHEVRIER S. (2007) - Les dépôts métalliques du Hallstatt D1 dans le Bassin parisien, in : Ph. Barral, A. Daubigney, C. Dunning, G. Kaenel, M.-J. Roulière-Lambert (dir.), L'âge du Fer dans l'arc jurassien et ses marges. Dépôts, lieux sacrés et territorialité à l'âge du Fer, actes du XXIXe colloque international de l'AFEAF (Bienne, 5-8 mai 2005), Besançon, Presses universitaires de Franche-Comté, 2007, p. 659-670.

COFFYN A., GOMEZ de SOTO J., MOHEN J.-P. (1981) - L'Apogée du Bronze atlantique, le dépôt de Vénat, Paris, Picard (L'Âge du Bronze en France, 1), 239 p.

COOMBS D. (1992) - Flag Fen Platform and Fengate Power Station Post Alignment - the Metalwork, Antiquity, 66, p. 504-517.

COONEY J., JOY J., ROBERTS B. (2011) - Collapsing Time. Understanding the Salisbury Hoard, Bulletin de l'Association pour la Promotion de l'Âge du Bronze, 8, p. 46.

CORDIER G. (1996) - Le dépôt du Bronze final du Petit-Villatte à Neuvy-sur-Barangeon (Cher) et son contexte régional, Joué-lès-Tours, chez l'auteur, 99 p.

CORDIER G. (2009) - L'âge du Bronze dans les pays de la Loire moyenne, Tours, La Simarre, $702 \mathrm{p}$.

CORDIER G., GRUET M. (1975) - L'Âge du Bronze et le Premier Âge du Fer en Anjou, Gallia-Préhistoire, 18, p. 157-287.

CUNLIFFE C., O'CONNOR B. (1979) - The Late Bronze Age Hoard from Danebury, Hants., in C. Burgess, D. Coombs (dir.), Bronze Age Hoards. Some Finds Old and New, Oxford (British Archaeological Reports, British Series 67), p. 235-244.

DAUTANT A., LESCA-SEIGNE A., SEIGNE J. (1984) - Sépulture à incinération d'un couple à Biganos (Gironde), in J. Gomez de Soto (dir.), Aspects des âges du Fer en CentreOuest, Angoulême, Musée, p. 43-46.

DÉCHELETTE J. (1910a) - Manuel d'Archéologie préhistorique, celtique et gallo-romaine, t. II, lèrepartie : Âge du Bronze, Picard, Paris, $512 \mathrm{p}$.

DÉCHELETTE J. (1910b) - Manuel d'Archéologie préhistorique, celtique et gallo-romaine, t. II, Archéologie celtique ou protohistorique. Appendices, Paris, Picard, $191 \mathrm{p}$.

DOIZE R. L. (1965) - La cachette de l'âge du Bronze de Chamery (Marne), Congrèspréhistorique de France, XVI, Monaco, 28 août - 5 septembre 1959 (Paris, SPF, 
1965), p. 530-538.

ELUÈRE C. (1979) - Le dépôt de bronzes de Maintenon (Eure-et-Loir) et les haches à douille à décor de nervures verticales de types britanniques, Bulletin de la Société préhistorique française, 76, 4, p. 119-128.

ELUÈRE C. (1982) - Les ors préhistoriques, Paris, Picard (l'Âge du Bronze en France, 2), $287 \mathrm{p}$.

FAVRAUD A. (1893) - A travers l'histoire de la contrée - Le trésor de Vénat, L'Avenir de laCharente, non paginé.

FILY M. (2008) - Les monuments funéraires et les dépôts métalliques dans le paysage rituel de l'âge du Bronze: l'exemple du centre-ouest de la Bretagne et du Finistère littoral (France), thèse de doctorat, université de Rennes 1, 621 p.

FILY M. (2009) - Les dépôts de la fin de l'âge du Bronze final de Kergaradec à Gouesnac'h (Finistère) : note préliminaire sur une découverte récente de l'horizon métallique de l'épée en langue de carpe, Bulletin de la Société préhistorique française, 106, 1, p. 95-107.

GACHINA J. (1975) - Les deux bracelets en bronze à terminaisons « en boules » de SaintJames à Port-d'Envaux (Charente-Maritime), Bulletin de la Société préhistorique française, 72,8, p. 253-255.

GASCÓ J. (2013) - La cache de l'âge du Fer de Roucadour (Thémines, Lot), in S. Verger et L. Pernet, Une Odyssée gauloise. Parures de femmes à l'origine des premiers échanges entre la Grèce et la Gaule, Arles, Errance (Archéologie de Montpellier Agglomération, 4), p. 169173.

GEORGE J., CHAUVET G. (1894) - La cachette de fondeur de Vénat, suivi de : Une cachette d'objets en bronze trouvée à Vénat, commune de Saint-Yrieix, près d'Angoulême, Bulletin et mémoiresde la Sociétéarchéologique et historique de la Charente, p. 105-343.

GERMOND G., CHAMPEME J.-M. (1998) - Un dépôt de l'Âge du Bronze final à Tourtenay (Deux-Sèvres), Bulletin de la Société préhistorique française, 95, 2, p. 255-268.

GIOT P.-R. (1954) - Quelques découvertes finistériennes de l’Âge du Bronze récent, Bulletin de laSociété archéologique du Finistère, LXXX, p. 55-61.

GIOT P.-R., avec coll. DAIRE M.-Y., MORZADEC H. (1995) - L'âge du Fer, Protohistoire dela Bretagne, Rennes, Ouest-France, p. 203-365.

GIRAUD P., NIVEZ E. (2014) - La nécropole de Verson «Le Mesnil», Bulletin de l'Association française pour l'étude de l'âge du Fer, 32, p. 51-54.

GOMEZ de SOTO J. (1980) - Les Cultures de l'Âge du Bronze dans le Bassin de la Charente, Fanlac, Périgueux, 119 p. 
GOMEZ de SOTO J. (1984) - Du Bronze final au Premier Âge du Fer dans le Bassin de la Charente, Transition Bronze final-Hallstatt ancien (colloque du 109ème Congrès National des Sociétés Savantes, Dijon, 1984), Paris, CTHS, p. 251-259.

GOMEZ de SOTO J. (2014) - Des éléments du Hallstatt C dans les derniers dépôts français de l'horizon métallique de l'épée en langue de carpe ? Un examen critique, Bulletin de la Société préhistorique française, 111, 4, p. 727-738.

GOMEZ de SOTO J. [coord.], BOURHIS J.-R., GHESQUIERE E., MARCIGNY C., MENEZ Y., RIVALLAIN J., VERRON G. (2009) - Pour en finir avec le Bronze final ? Les haches à douille de type armoricain en France, in M.-J. Roulière-Lambert, A. Daubigney, P.Y. Milcent, M. Talon, J. Vital (éd.), De l'âge du Bronze à l'âge du Fer en Europe occidentale (Xe - VIIe siècle av. J.-C.). La moyenne vallée du Rhône aux âges du Fer (actes du XXXe colloque international de l'A.F.E.A.F., Saint-Romain-en-Gal, 26-28 mai 2006), Dijon, Revuearchéologique de l'Est, $27^{\mathrm{e}}$ suppl., p. 507-512.

GOMEZ de SOTO J., MILCENT P.-Y. (2000) - De la Méditerranée à l'Atlantique : échanges et affinités culturelles entre le nord-ouest et le sud-ouest de la France de la fin du Xème au Vème s. av. J.-C., in T. Janin T. (dir.), Mailhac et le Premier Age du Fer en Europe occidentale. Hommages à Odette et Jean Taffanel, actes du colloque en hommage à Odette et Jean Taffanel (Carcassonne, 17-20 sept. 1997), Lattes (Monographies d'Archéologie méditerranéenne, 7), p. 350-371.

GUILAINE J. (1969) - Le dépôt de bronzes de Carcassonne, Revue archéologique de Narbonnaise, II, p. 1-45.

GUILAINE J. (1972) - L'Âge du Bronze en Languedoc occidental, Roussillon, Ariège, Paris, Klincksiek (Mémoires de la Société préhistorique française, 9), 460 p.

HAWKES J. (1937) - The Archaeology of the Channel Islands. I. The Bailiwick of Jersey, Jersey, Société jersiaise, $319 \mathrm{p}$.

HINGUANT S., LE GOFF E., avec coll. GEBHARDT A., GRALL B., MAGUER P., MARGUERIE D. (1998) - Un site de l'Âge du Fer stratifié en milieu rural : l'établissement de Keralio à Pont-l'Abbé (Finistère). Revue archéologique de l'Ouest, 15, p. 59-114.

JAHIER I. (1999) - Parures d'une sépulture féminine de Courseulles-sur-Mer (Calvados), Nos ancêtres les Gaulois aux marges del'Armorique, Nantes, Musée Dobrée, p. 138-139.

JOCKENHÖVEL A. (1975) - Zum Beginn der Jungbronzezeitkultur in Westeuropa, Jahresbericht des Institut für Vorgeschichte der Universität Frankfurt am Main, p. 134-181.

JOCKENHÖVEL A. (1980) - Die Rasiermesser in Westeuropa, C.H.Beck'sche Verlag., Münich (Prähistorische Bronzefunde, VIII, 3), 238 p.

KENDRICK T. D. (1928) - The Archaeology of the Channel Islands. I. The Bailiwick of Guernsey, Londres, Methuen \& $\mathrm{C}^{\circ}, 273 \mathrm{p}$.

KIBBERT K. (1984) - Die Äxte und Beile in Westdeutschland II, C. H. Beck Verlag, Münich (Prähistorische Bronzefunde, IX, 13), 262 p. 
KOLLING A. (1968) -Späte Bronzezeit an Saar und Mosel, Bonn, Habelt, t. 1, 221 p., t. 2, 69 $\mathrm{pl}$.

LAROCHE M., AMBERT P., FIGUERO-LARRE V., KLEMM V., ROVIRA LLORENS S., PRANGE M. (2008) - Le Launacien dans le sud de la France : l'exemple du dépôt de Bautarès (Fontès, dép. Hérault) et ses relations avec le district minier de Cabrières-Péret, Archäologisches Korrespondenzblatt, 38,1, p. 45-59.

LAVALLÉE L. (1888) - Compte-rendu de la séance du 8 nov. 1888, Bulletin et mémoires de la Sociétéarchéologique du département d'Ille-et-Vilaine, XVIII, p. LXXXV-LXXXVI.

LAVALLÉE L. (1891) - Compte-rendu de la séance du 12 mars 1889, Bulletin et mémoires de la Sociétéarchéologique du département d'Ille-et-Vilaine, XXI, p. IX-X.

LE CARLIER C., LE BANNIER J.-C., ARANDA B., MARCIGNY C. (2009) -Analyses chimiques des objets de trois dépôts normands (Trelly, Agneaux, Surtainville) : variation au sein de chaque dépôt, intercomparaison,Séminaires archéologiques de l'Ouest, 2 avril 2009 (non publié).

LE CARLIER C., LE BANNIER J.-C., MARCIGNY C., FILY M., MELIN M. (2011) L'analyse des objets à base cuivre protohistoriques de l'Ouest de la France. L'importance de la représentativité des analyses à l'échelle régionale, Journée $d u$ "CReAAH "Archéologie, Archéosciences, Histoire (Rennes, 2 avril 2011), p. 48.

LE MEN R. G. (1860) - Celtic Arms and Ornaments Found at Ploneour, Brittanny, ArchaeologiaCambrensis, III'série, VI, p. 136-139.

LETOR A. (1999) - Problèmes de méthodologie lors de l'étude d'objets muséaux anciens : le cas des haches à douille armoricaines de Belgique, Anthropologie et Préhistoire, 110, p. 107117.

L'HELGOUACH J. (1985) - Ruffigné, La Forgerais, in: Informations archéologiques. Circonscription des Pays de la Loire, Gallia-Préhistoire, 28, 2, p. 376-377.

L'HELGOUACH J. (1999) - Fragment du dépôt de haches à douille de Ruffigné (LoireAtlantique), Nos ancêtres les Gaulois aux marges del'Armorique, Nantes, Musée Dobrée, p. 18-19.

LUKIS F. C. (1843) - On the Antiquities of Alderney, Journal of the British ArchaeologicalAssociation, III, p. 1-15.

MAGGI C., PAITIER H. (1994) - Dépôt du Bronze final de la Tiédenaie à Saint-Père-en-Retz (Loire-Atlantique), Revue archéologique de l'Ouest, 11, p. 119-130.

MARCHADIER E., avec coll. BOUCHET J.-M., BURNEZ C., GOMEZ de SOTO J. (2005) Premier âge du Fer en Saintonge et Aunis. Etude typo-chronologique du mobilier céramique,Saintes, Société d'Archéologie et d'Histoire de la Charente-Maritime (Recherches archéologiques en Saintonge et Aunis, 17), 155 p. 
MARIËN M.-E. (1958) - Trouvailles du Champ d'Urnes et des Tombelles hallstattiennes de Court-Saint-Etienne, Bruxelles, Musées royaux d'Art et d'Histoire, 269 p.

MENEZ Y., GOMEZ de SOTO J. (2006) - L'habitat de Kergariou à Quimper, Bulletin de l'Association française pour l'Étude de l'âge du Fer, 24, 2006, p. 83-85.

MENEZ Y., GOMEZ de SOTO J., DUPRÉ M. (2005) - Quimper - Finistère. L'habitat de l'âge du Fer de Kergariou, rapport d'opération archéologique, Rennes, Service régional de l'archéologie de Bretagne, $36 \mathrm{p}$.

MENEZ Y. et FILY M. (2009) - Hengoat (Côtes d'Armor). Note sur les modalités de découverte du Dépôt de haches à douille du Moulin de Bizien, rapport, Rennes, Service régional de l'Archéologie, $11 \mathrm{p}$.

MILCENT, P.-Y. (1993) - L'Âge du Fer en Armorique à travers les ensembles funéraires (IXe-IIIe siècles avant J.-C.), Antiquités nationales, 25, p. 17-50.

MILCENT P.-Y. (2004) - Le premier âge du Fer en France centrale. Paris, Société préhistorique française (Mémoire, XXXIV), $718 \mathrm{p}$.

MILCENT P.-Y. (2012) - Le temps des élites en Gaule atlantique. Chronologie des mobiliers et rythmes de constitution des dépôts métalliques dans le contexte européen (XIIIe-VIIe s. av. J.-C.), Rennes, Presses universitaires, $253 \mathrm{p}$.

MILCENT P.-Y. (2103a) - Le dépôt de la Mouleyre à Saint-Pierre-d'Eyrac (Haute-Loire), in S. Verger et L. Pernet (dir.), Une Odyssée gauloise. Parures de femmes à l'origine des premiers échanges entre la Grèce et la Gaule, Arles, Errance (Archéologie de Montpellier Agglomération, 4), p. 166-168.

MILCENT P.-Y. (2013b) - Le dépôt de Lavoûte-Chillac (Haute-Loire), in Verger S. et Pernet L., Une Odyssée gauloise. Parures de femmes à l'origine des premiers échanges entre la Grèce et la Gaule, Arles, Errance (Archéologie de Montpellier Agglomération, 4), p. 168.

MILLOTTE J.-P. (1972) - La cachette de la Mouleyre à Saint-Pierre-d'Eyrac (Haute-Loire), les tumulus de Chavéria (Jura) et les débuts du premier âge du Fer en France, Congrès préhistorique de France (XIXe session, Auvergne, 1969), p. 293-298 (avec appendices par R. Gounot et J.-P. Daugas, p. 299-301).

MONGELLAZ J., MEURET J.-C. (1999) - Ensemble ( ?) de deux anneaux de cheville à oves creux, deux bracelets à bossettes pleines et deux haches à talon, Nos ancêtres les Gaulois aux marges del'Armorique, Nantes, Musée Dobrée, p. 127.

de MORTILLET G., de MORTILLET A. (1881) - Musée préhistorique, Paris, Reinwald, 100 pl.

NICKELS A., avec coll. MARCHAND G. et SCHWALLER M., 1989. Agde. La nécropole du premier âge duFer, Paris (Revue archéologique de Narbonnaise, suppl. 19), 499 p.

O'CONNOR B. (1980) - Cross-Channel Relations in the Later Bronze Age. Relations Between Britain, North-Eastern France and the Lower Countries During the Later Bronze 
Age and the Early Iron Age, with Particular Reference to the Metalwork, Oxford, BAR International Series 91, 858 p.

O'CONNOR B. (2007) - Llyn Fawr metalwork in Britain: a Review, in C. Haselgrove, R. Poper (dir.), The Earlier Iron Age in Britain and the Near Continent, Oxford, Oxbow Books, p. 64-79.

OGES L. (1952) - Une cachette de fondeur vieille de 3.000 ans découverte à Plobannalec, LeTélégramme de Brest, édition du 31 mars.

PIETTE J. (1989) - Le premier âge du fer dans l'Aube, découvertes inédites ou peu connues. Pré-et Protohistoire de l'Aube, Voipreux, Association régionale pour la protection et l'étude du patrimoine préhistorique, p. 229-241.

PHILIPPE A. (1992) - Le dépôt de haches à douille armoricaines de La Forgerais, en Ruffigné (L.-A.), étude descriptive et comparative, mémoire de maîtrise, université de Nantes.

POSTMA H., FONTIJN D., SCHILEBEECKX P., PEREGO R. C., BUTLER J. J. (2005) Neutron Resonance Capture Analysis of Geistingen Axes, Lunula. Archaeologia protohistorica, XIII, p. 41-46.

RIVALLAIN J. (1971) - Contribution à l'Etude du Bronze Final en Armorique. Elaboration d'une méthode appliquée aux dépôts de haches à douille armoricaines, Rennes, Université de Haute Bretagne, $146 \mathrm{p}$

RIVALLAIN J. (2012) - Les haches à douille armoricaines revisitées. Apports des travaux et des études de la deuxième moitié du XXe siècle en Bretagne, Alet (Dossiers du Centre régional d'Archéologie d'Alet, suppl. A1), 224 p., 1 cédérom.

ROBERT C. (1898) - Le menhir de Dol et sa légende, Bulletin archéologique de l'Association bretonne publié par la classe d'Archéologie, $3^{e}$ série, actes du $38^{e}$ congrès tenu à Rennes les 23-29 mai 1897, Saint-Brieuc, 1898, p. 58-66.

ROUSSOT-LARROQUE J. (1982) - Pour un musée imaginaire. Documents inédits des archives de la Société Archéologique de Bordeaux, Bulletin et mémoires de la Société archéologique de Bordeaux, LXXIII, p. 21-48.

ROUSSOT-LARROQUE J. (1984) - Le dépôt de Césarin à Tulle et les relations est-ouest à la fin de l'Age du Bronze, Élémentsde Pré et Protohistoire européenne. Hommages à J.-P. Millotte, Paris, Les Belles Lettres, p. 617-627.

ROUSSOT-LARROQUE J. (2005) - Bordeaux préhistorique. Les racines de Bordeaux, du Néolithique à la fin de l'âge du Bronze, Revue archéologique de Bordeaux, XCVI, p. 3-36.

ROUSSOT-LARROQUE J. (2013) - L'épée et le rasoir : la transition Bronze-Fer autour de l'estuaire de la Gironde, in Colin A. et Verdin F. (dir.), L'âge du Fer en Aquitaine et sur ses marges. Mobilité des hommes, diffusion des idées, circulation des biens dans l'espace européen à l'âge du Fer, Bordeaux (Aquitania, suppl. 30), p. 57-81. 
ROWLANDS M. J. (1976) - The Organisation of Middle Bronze Age Metalworking, Oxford (British Archaeological Reports, 31), 446 p.

ROY E. (2000) - Pont L'Abbé (Finistère), fouille archéologique avant l'aménagement d'unlotissement au lieu-dit Kerarthur, document final de synthèse, Rennes, AFAN et SRA de Bretagne.

SAN JUAN G. (1999) - Mobilier de la fosse sépulcrale F 20 de Basly (Calvados), La Campagne, Nos ancêtres les Gaulois aux marges del'Armorique, Nantes, Musée Dobrée, p. 135.

SAN JUAN G., LE GOFF I. (2003) - La nécropole du VIe siècle avant J.-C. de «La Campagne » à Basly (Calvados), Les marges de l'Armorique à l'âge du Fer, archéologie et histoire: culturematérielle et sources écrites, actes du XXIIIe colloque international de l'AFEAF (Nantes, 1999), Rennes (Revue archéologique de l'Ouest, suppl. 10), p. 59-102.

STEAD I. M. (1998) - The Salisbury Hoard, Londres, Tempus Publishing, 160 p.

TABBAGH A., VERRON G. (1983) - Etude par prospection électromagnétique de trois sites à dépôts de l'Âge du Bronze, Bulletin de la Société préhistorique française, 80, 10-12, p. 375389.

TALON M. (2012) - Épingle de type Picardie, in A. Lehoërff (dir.), Par-delà l'horizon. Sociétés en Manche et mer du Nord, il y a 3500 ans, Paris, Somogy, p. 110.

TAUVEL D. (1974) - Le premier Âge du Fer dans la Vienne. $2^{\mathrm{e}}$ partie, Revue archéologique duCentre, XIII, 1-2, p. 3-24.

VERGER S. (1999) - Epée à languette, épée à antennes, Nos ancêtres les Gauloisaux portes de l'Armorique, Nantes, Musée Dobrée, notice 211, p. 113.

VERGER S. (2013) - Les dépôts launaciens, in S. Verger et L. Pernet (dir.), Une Odyssée gauloise. Parures de femmes à l'origine des premiers échanges entre la Grèce et la Gaule, Arles, Errance (Archéologie de Montpellier Agglomération, 4), p. 100-118.

VERNEY A. (1990) - Le dépôt de Challans (Vendée), Bulletin de la Société préhistorique française, 87, 10-12, p. 396-417.

VERNEY A. (1993) - Les nécropoles de l'Âge du Fer en Basse-Normandie. Bilan de trois siècles de découvertes, Les Celtes en Normandie. Les rites funéraires en Gaule (IIIe - Ier siècleavant J.-C., actes du XIVe colloque de l'Association française pour l'étude de l'Âge du Fer (Évreux, mai 1990), Rennes (Revue archéologique de l'Ouest, suppl. 6), p. 95-113.

VERNEY A. (1994) - La collection préhistorique du Musée d'Évreux, Évreux (Études et documents du Musée, 1), $52 \mathrm{p}$.

VERNEY A. (1999) - Dépôt de Trelly (Manche), Nos ancêtres les Gaulois aux marges del'Armorique, Nantes, Musée Dobrée, notice 4, p. 20. 
VIEAU M. (1976) - Étude de collections d'objets de l'âge du Bronze du Museum d'Histoire naturelle de Nantes, du musée du château de Noirmoutier, du musée de Châteaubriant, Etudespréhistoriques et protohistoriques. Pays de la Loire, 4, p. 1-124.

VILLES A. (1995) - Actualité des recherches sur l'âge du Fer entre l'Yonne et la Marne, Fastes desCeltes anciens, Troyes et Nogent-sur-Marne, éd. des musées, p. 5-35 (dépôt de Crancey : ibid., p. 130-131).

WARMENBOL E. (2012-2013) - La hache à douille « de Nismes » (Viroinval, Province de Namur). Quelques réflexions autour des haches armoricaines avec une provenance belge, Archéo-Situla, 32-33, p. 3-8.

José GOMEZ de SOTO

Directeur de recherche émérite au CNRS

UMR 6566 «CReAAH, Centre de Recherche en Archéologie, Archéosciences, Histoire » Laboratoire Préhistoire et Archéosciences,

Bât. 24-25, Université de Rennes 1, campus de Beaulieu, 35042 Rennes cedex jgzdsoto@free.fr 